\title{
The Contribution of Premotor Cortico-Striatal Projections to the Execution of Serial Order Sequences
}

\author{
Asai Sánchez-Fuentes, Kathia I. Ramírez-Armenta, Anil Kumar Verma-Rodríguez, \\ Edgar Díaz-Hernández, Antonio Aguilar-Palomares, Josué O. Ramírez-Jarquín, and \\ DFatuel Tecuapetla
}

https://doi.org/10.1523/ENEURO.0173-21.2021

Instituto de Fisiología Celular, Universidad Nacional Autónoma de México, Ciudad de México 04510, México

\begin{abstract}
Striatal activity is necessary to initiate and execute sequences of actions. The main excitatory input to the striatum comes from the cortex. While it is hypothesized that motor and premotor cortico-striatal projections are important to guide striatal activity during the execution of sequences of actions, technical limitations have made this challenging to address. Here, we implemented a task in mice that allows for the study of different moments to execute a serial order sequence consisting of two subsequences of actions. Using this task, we performed electrophysiological recordings in the premotor (M2) and primary motor (M1) cortices, and statedependent optogenetic inhibitions of their cortico-striatal projections. We show that while both M2 and M1 contain activity modulations related to the execution of self-paced sequences, mainly, the premotor corticostriatal projections contribute to the proper execution/structuring of these sequences.
\end{abstract}

Key words: action sequences; cortico-striatal; motor cortex; optogenetics; premotor cortex; synapses

\section{Significance Statement}

It is currently hypothesized that synapses from the primary motor (M1) and premotor (M2) cortices that innervate the striatum may guide the proper execution of sequences. Here, we evaluated this hypothesis by training animals to execute self-paced sequences: performing recordings in M2-M1 or manipulating their cortico-striatal projections during the execution of these sequences. We show that both, M2-M1 corticostriatal projections contribute to sequence initiation, however sequence execution is predominantly influenced by M2. Remarkably the contribution of the cortico-striatal projections from M2 is mainly before the initiation of the sequence working to sustain the structure of the sequences, mainly during the beginning. These findings may have implications for pathologic conditions where the self-paced generation of sequences of actions is impaired.

\section{Introduction}

In everyday life, we continuously move between sequences of motor actions. One of the main proposed drivers involved in the learning and execution of motor sequences are cortico-striatal projections. The study of action sequences in relation to cortico-striatal function has become increasingly important since the discovery

Received April 17, 2021; accepted August 8, 2021; First published August 31, 2021.

The authors declare no competing financial interests. that symptoms in patients with Parkinson's disease and obsessive-compulsive disorder maybe be caused by disruptions to cortico-striatal projections (Graybiel, 1998; Redgrave et al., 2010; Burguière et al., 2013).

The striatum, the primary input to basal ganglia (BG), is a subcortical structure whose activity is necessary to initiate and execute a sequence of actions (Cui et al., 2013;

Author contributions: A.S.-F. and F.T. designed research; A.S.-F., K.I.R.-A., E.D.-H., A.A.-P., and J.O.R.-J. performed research; A.S.-F., K.I.R.-A., A.K.V.-R., E.D.-H., and A.A.-P. analyzed data; A.S.-F. and F.T. wrote the paper. 
Jin et al., 2014; Rothwell et al., 2015; Tecuapetla et al., 2016; Dhawale et al., 2021). Recent evidence suggests that a specific subcircuit within the BG, the indirect pathway, is essential for the transition between subsequences (Geddes et al., 2018; Tecuapetla et al., 2016).

The striatum's main glutamatergic inputs come from the cortex and the thalamus (Wilson, 1989). Several studies suggest that the cortical inputs are essential to execute motor sequences (Tanji and Evarts, 1976; Inase et al., 1996; Martiros et al., 2018; Shima and Tanji, 2000; Fujii and Graybiel, 2005; Shima et al., 2007; Burguière et al., 2013; Smith and Graybiel, 2013; Friedman et al., 2015; Rothwell et al., 2015; Kupferschmidt et al., 2017; Martiros et al., 2018). However, the specific contribution of corticostriatal projections to the execution of self-paced action sequences remains unclear.

To date, it is known that the supplementary motor area in primates (SMA), which corresponds to the secondary motor cortex (M2) in rodents, is active before starting a sequence of actions (Donoghue and Wise, 1982; Romo and Shultz 1987; Passingham et al., 1988; Shima et al., 2007; Nakayama et al., 2008). SMA activity is important to adapt the behavior in response to contingency changes (block changes; Hikosaka and Isoda, 2010; Siniscalchi et al., 2016). These findings have led to the hypothesis that the premotor cortex guides the striatal activity to initiate and execute action sequences. In rodents, a decrease in the activity of neurons in M2 decreases the probability of alternating between two actions (Rothwell et al., 2015). Strikingly however, studies using cortical lesions suggest that the striatum (BG) can control the execution of a sequence of actions independently from the cortex (Kawai et al., 2015; Dhawale et al., 2021). Therefore, we implemented a self-paced serial order sequence task that allows for probing the contribution of M2 cortico-striatal projections during the initiation, execution, and transition between subsequences of actions. By recording neuronal activity in premotor (M2) and primary motor (M1) cortices and performing time-dependent optogenetic inhibitions of the cortico-striatal projections, we identified specific contributions of the premotor cortico-striatal projections to the execution of self-paced

This work was supported by the Ciencia Basica Consejo Nacional de Ciencia y Tecnología (CONACyT) Grant 220412; Fronteras de la Ciencia CONACyT Grants 2022 and 154039; Dirección General de Asuntos del Personal Académico - Programa de Apoyo a Proyectos de Investigación e Inovación Tecnológica (DGAPA-PAPIIT)-Universidad Nacional Autónoma de México (UNAM) Grants IA200815, IN226517, and IN203420; and the Catedra Marcos Moshinsky 2019 (F.T.). A.S.-F. is a doctoral student from the Programa de Doctorado en Ciencias Biomédicas, UNAM and has received the CONACyT Fellowship 597738.

Acknowledgements: We thank Professor Rui Costa for the Emx1-cre mice, Dr. E. Galarraga for electrophysiology ex vivo support, Dr. Y. Ramiro-Cortes for imaging support, and Dr. Gabriela J. Martins and Dr. Anna Hobbiss for proofreading the English of this manuscript.

Correspondence should be addressed to Fatuel Tecuapetla at fatuel@ifc. unam.mx.

https://doi.org/10.1523/ENEURO.0173-21.2021

Copyright (C) 2021 Sánchez-Fuentes et al.

This is an open-access article distributed under the terms of the Creative Commons Attribution 4.0 International license, which permits unrestricted use, distribution and reproduction in any medium provided that the original work is properly attributed. serial order action sequences. Our results support a model in which the cortico-striatal terminals from M2 guide the appropriate execution of self-paced sequences of actions.

\section{Materials and Methods}

\section{Animals}

The institutional committee of the Cell Physiology Institute, at the National Autonomous University of Mexico, approved all procedures for the care and use of laboratory animals (protocol number FT121-17). This protocol follows the National Norm for Animals' use (NOM062-ZOO-1999). Male and female mice from two to three months of age at the start of experiments were used for this study. Two genotypes were used: C57BL/6J (The Jackson Laboratory, RRID: IMSR_JAX_000664) or Emx1Cre mice (targeting the Cre recombinase expression in pyramidal cortical neurons), which had been backcrossed into C57BL/6J for at least six generations (Gorski et al., 2002). Emx1-Cre parental line was donated by Professor Rui M. Costa from the Champalimaud Center for the Unknown (RRID: MGI_5141283). All animals were obtained from our breeding colony in our institutional bioterium (the Emx1-Cre line is maintained in heterozygosis). Animals are housed under a 12/12 h light/dark cycle (lights on at 6 A.M.) with ad libitum access to food and water before beginning behavioral experiments.

\section{Training}

We used operant conditioning boxes equipped with two retractable levers to implement forced and self-paced serial order sequences in mice $(21.6 \mathrm{~cm}$ long $\times 17.8 \mathrm{~cm}$ wide $\times 12.7 \mathrm{~cm}$ high; Med-Associates, catalog \#MED$307 W-D 1)$. One lever was positioned on the left panel (subsequence 1; S1), and the other was located on the front panel (subsequence 2; S2) on the left side of the magazine (Fig. 1B,C). A small sugar pellet, $14 \mathrm{mg}$ (BioServ, catalog \#F05684), was delivered as a reward in the magazine. Entries to the magazine were registered with an infrared beam. A second infrared beam was positioned between the magazine and left lever press (Fig. 1B,C) to calculate the latency to start the sequences of actions. Mice were subjected to food restriction throughout training and given enough food after daily training sessions to keep them at $80-85 \%$ of their original weight, depending on performance.

For the first exposure to the operant box, animals were placed in the box without levers for $30 \mathrm{~min}$. A total of 30 pellets were delivered individually at random intervals (on average every $60 \mathrm{~s}$ ). Over the next $3 \mathrm{~d}$, the animals were presented with lever 1 or lever 2 and received a reward each time they pressed the lever. After eight rewards, lever 1 was retracted, and lever 2 was presented. The session finished once the animal got 16 pellets, or $30 \mathrm{~min}$ had passed. Afterward, the training schedule changed to $3 \mathrm{~d}$ at a fixed ratio eight (FR8) schedule on each lever, whereby animals had to accumulate eight lever presses to receive a reward and retract the lever, followed by a second lever presentation, which also required 8 presses to 
A

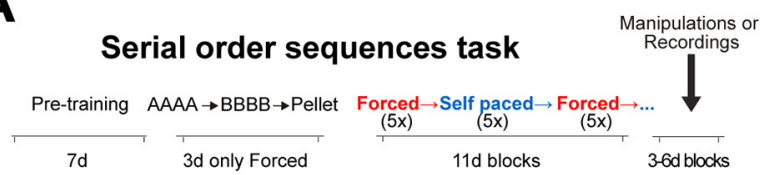

B

B Forced Sequence (Levers are exposed-retracted)
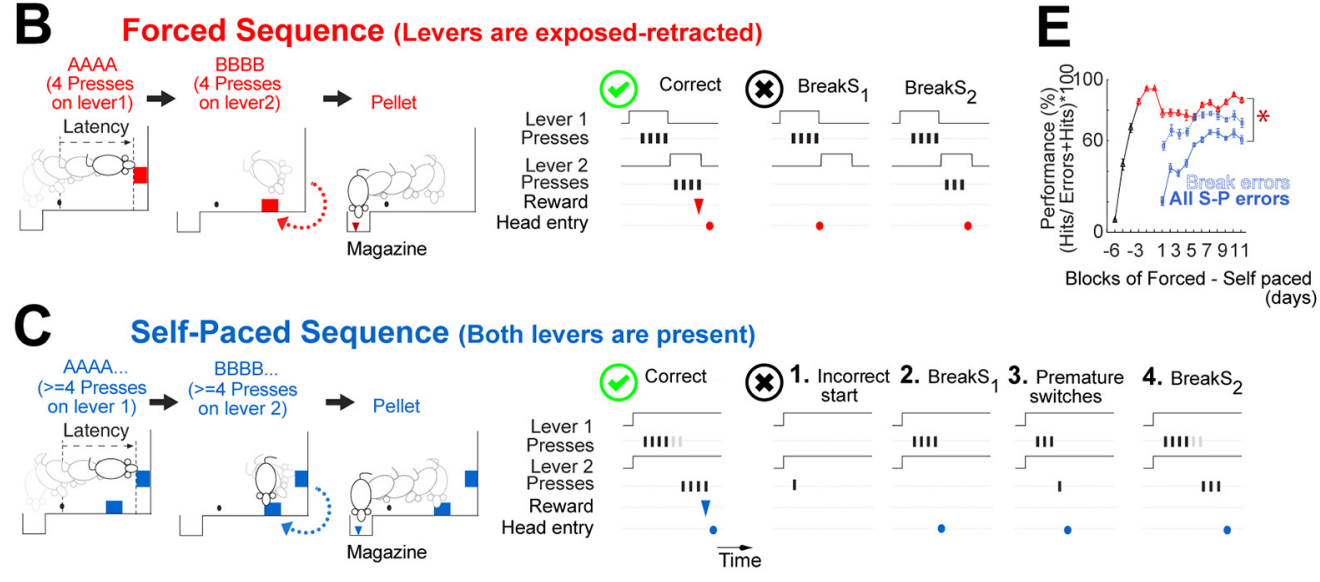
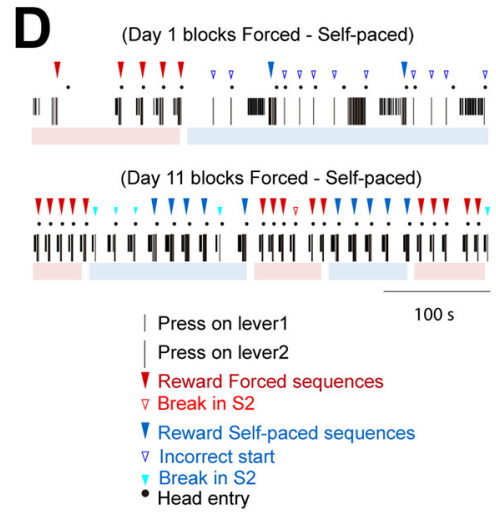

$\mathbf{G} \circledast$
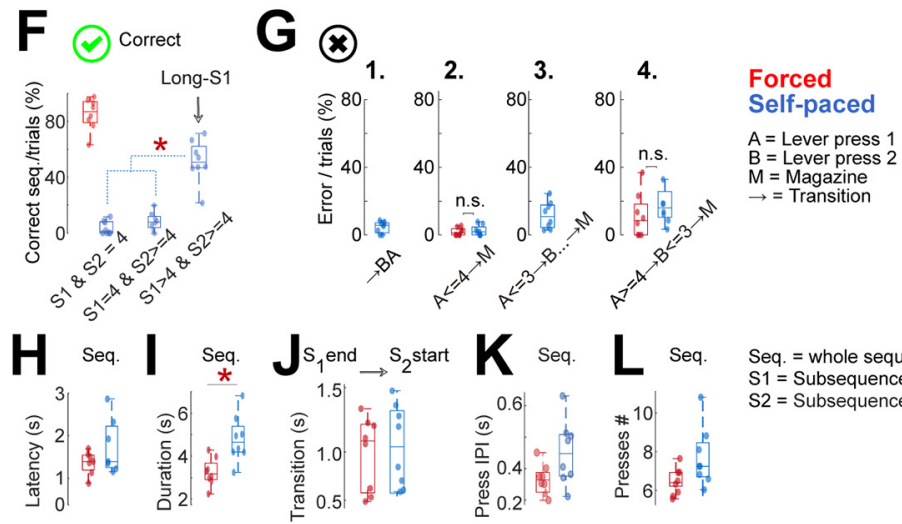

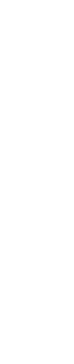

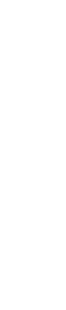


trained to do forced serial order sequences $(3 \mathrm{~d})$. Here, animals were presented with a lever 1 ; they had to do four continuous lever presses for the lever to be retracted, followed by extension of a second lever (lever 2). Animals had to do four continuous lever presses on lever 2 for it to be retracted, and a reward was delivered into the magazine (Fig. 1B). If the animals executed fewer than four lever presses on lever 1 or lever 2 by visiting the magazine, a time out (10 s) was presented. The session finished when mice got 30 rewards, or $30 \mathrm{~min}$ had passed. After these $3 \mathrm{~d}$ of forced sequences, the animals entered the last stage of training: blocks of forced-self-paced sequence sessions. These sessions began with a block of forced trials, switching to a block of self-paced trials in the same session. The switch between blocks was conditioned by achieving five consecutive correct sequences. For the self-paced sequences, the animals were presented with the two levers at the same time. They had to do at least four presses on lever 1 followed by at least four presses on lever 2, to obtain a reward in the magazine. Unlike in forced blocks, in self-paced blocks the animals decided when to execute the transition between the subsequences of presses from lever 1 to lever 2 (Fig. 1C). Upon finishing a self-paced block, both levers are retracted for $3 \mathrm{~s}$ for an intertrial interval before starting a new forced block. If the animals executed fewer than four presses on lever 1 or lever 2 by visiting the magazine, a time out (10 s) was presented. The session finished once the animals received 70 rewards ( 35 forced and 35 selfpaced rewards) or $30 \mathrm{~min}$ had passed. The block sessions continued for 11-13d until a stable performance was reached; see Fig. 1E). All timestamps were recorded with a resolution of $10 \mathrm{~ms}$ with the Med-PC IV software suite (Med-Associates).

\section{Single-unit recording and antidromic photo- identification}

To record the cortical activity, either a fixed or movable 16electrode array [tungsten ( $35 \mu \mathrm{m}$; Innovative Neurophysiology) was implanted]. The neurons' spikes were sorted online (Central software, Blackrock Microsystems), and clear waveforms with a signal-to-noise ratio $>3: 1$ were used for further analysis using offline-sorting (Offline Sorter, Plexon Inc.). To define whether a recorded unit projected to the striatum, we used in vivo antidromic photo-identification (Lima et al., 2009). In short, we injected ChR2 (UPENN-vector core catalog \#AV1-20298P) in the cortex of interest of Emx1-Cre animals. A movable electrode array was implanted $200 \mu \mathrm{m}$ above the ChR2 expression site during the same surgery, and an optic fiber (Thorlabs catalog \#CFLC230-10, FT200EMT) was implanted into the striatum (ipsilateral to injection). Using the electrode array, we could record activity of cortical neurons. At the end of the behavior session, we used a light stimulation (2 $\mathrm{mW}, 473 \mathrm{~nm}, 10 \mathrm{~Hz}, 1 \mathrm{~s}, 10-\mathrm{ms}$ pulses; Laserglow) delivered by the optic fiber while recording neuronal activity. This allowed us to verify whether the recorded neuron was antidromically photo-activated (Extended Data Fig. 3-2). The movable array allowed us to search for responding cells in at least five sessions per animal, advancing the array $100 \mu \mathrm{m} 24 \mathrm{~h}$ before the recording. Only units that responded to the light (i.e., presented a correlation of $>0.9$ between the behavioral spike and the antidromic spike and presented a latency to light $<10$ $\mathrm{ms}$ ) were considered.

\section{Per-trial rescaling of neural activity}

In both sequence types, forced and self-paced, each sequence had a slightly different durations and could not be directly averaged. To mitigate this, we employed a time rescaling procedure (Kobak et al., 2016), to evaluate each recorded unit's overall response pattern. Thus, we performed a rescaling of trials with at least four presses in each sequence, defining the following alignment events: first, second, penultimate, and last press of subsequence 1 and subsequence 2 . Spike trains were transformed to instantaneous firing rate by applying a Gaussian convolution $(\sigma 25 \mathrm{~ms})$ at $100 \mathrm{~Hz}$. The activity was rescaled through linear interpolation to the average interpress interval and inter sequence interval of all recorded animals' sessions.

\section{Analysis of the electrophysiological recordings in vivo}

Once a putative unit was isolated (through online and off-line sorting), the spikes' timestamps were aligned to the first lever press in the sequences of actions using custom-developed scripts in MATLAB (MathWorks).

$Z$ score test: to determine differences between the $Z$ score activity from $M 2$ versus $M 1$, we averaged the $z$ score activity in the same time window from the two indicated cells and calculated the difference in terms of $z$ score with the following equation:

$$
\text { Zdifference }=\frac{Z 1-Z 2}{\sqrt{\left(\frac{1}{N 1-3}\right)+\left(\frac{1}{N 2+3}\right)}},
$$

where $\mathrm{Z} 1$ is the average normalized activity of the M2 units and $Z 2$ for the M1; N1 is the sample of units from M2, and N2 the sample of units from M1 (Fig. 2J; Extended Data Fig. 2-1H). The results were compared in a normal distribution table to determine the corresponding $p$ value (Chen and Popovich, 2011).

\section{Regression analysis of the neuronal activity and behavior}

We made linear regression analysis to ask whether the firing rate in each bin of time was correlated with parameters of the task. For this purpose, we first aligned the spikes to each epoch (first, second, penultimate, and last lever press for each sequence) and took $5 \mathrm{~s}$ before and 5 $\mathrm{s}$ after each epoch. Spike trains were transformed to instantaneous firing rate as described above. We calculated the spike frequency using a 200-ms time window with 10ms steps. We separated the trials and sorted them by the number of lever presses in the sequences $(2,4,6$, up to 16 , grouped by every increment), for the sequence and transition duration (sorted by duration in descending manner, seven categories), for latency (grouped every one second, starting with $0.5 \mathrm{~s}$.). Then we made a regression analysis with permutation test in two manners. For the first, we used a bin of time of $200 \mathrm{~ms}$ with a sliding window of 

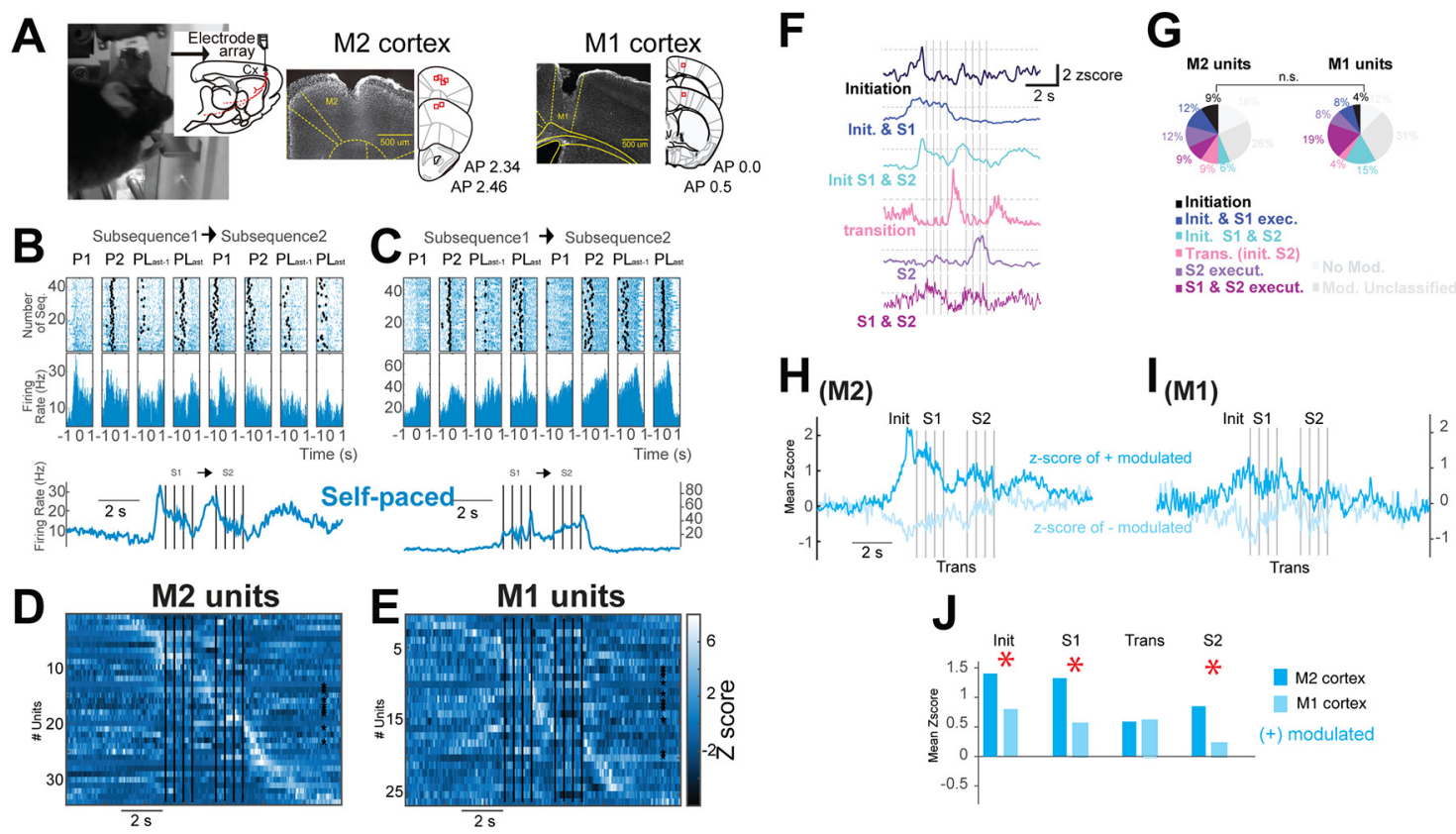

Figure 2. Activity modulation in premotor and motor cortical neurons during the execution of self-paced sequences of actions. $\boldsymbol{A}$, Image of a mouse while pressing one of the levers and photomicrographs of coronal sections of M2 (middle) or M1 cortex (right), illustrating the cannula/electrode array's tracks in the recorded sites. $\boldsymbol{B}, \boldsymbol{C}$, Raster plots and perievent histogram from an M2 or an M1 unit, respectively, aligned to the first (P1), second (P2), penultimate (PLast-1), and last lever press (PLast) of S1 and S2 for selfpaced sequences. Bottom panels, Mean firing rate from the upper panels. $\boldsymbol{D}, \boldsymbol{E}, \boldsymbol{Z}$ score of individual units. $\boldsymbol{F}$, Representative firing of different cells presented as $z$ score, illustrating the different categories of modulation during the execution of the sequences Init \& S1 execu = Initiation and S1 execution; Init. S1 \& S2 = Initiation of S1 and S2; Trans.(init. S2) = Transition or initiation S2; S2 execut. = S2 execution; S1 \& S2 execut. = S1 and S2 execution. $\mathbf{G}, \%$ of cells related to each category in $\boldsymbol{F}$. $\boldsymbol{H}, \boldsymbol{I}$, Mean $\boldsymbol{z}$ score from the units recorded that presented significant modulation $1 \mathrm{~s}$ before the initiation of the sequence from M2 or M1. $J$, Comparison of the mean $z$ score from $\boldsymbol{H}, \boldsymbol{I}$, M2 (dark blue) versus M1 (light blue) for positively modulated units; ${ }^{*} p<0.05, z$ score test. Init. = initiation, $S 1$ = subsequence 1, Trans = transition, and S2 = subsequence 2. Extended Data Figure 2-1 includes the activity modulation in premotor and motor cortical neurons during the execution of forced sequences of actions. n.s. $=p>0.05$.

$10 \mathrm{~ms}$ and ask whether the variable of interest was correlated with the firing rate (Fig. $3 A$; Extended Data Fig. $3-1 B$ ). For the second regressions analysis, we used specific windows of time: (1) mean firing rate $1 \mathrm{~s}$ before the start of the sequence; (2) mean of firing rate during the sequence; $(3,4$, and 5$)$ average of firing rate during $S 1$, firing rate during transition or firing rate during S2 (Fig. $3 B$ ). To resolve statistically whether the regression's $p$ value was significant, we ran 1000 permutations and divided the sum of times that the $p$ value $>p$ value initial between the number of permutations. Only regressions with $\beta$ coefficient different from zero $(p<0.05$ and $R>0.6)$ were accepted.

\section{ROC curve analyses and permutations}

To determine the percentage of modulated units along time in each epoch, we performed a ROC curve analysis to ask whether the spike frequency in each time bin was different from baseline time. We first aligned the spikes to each epoch (e.g., first press) using $4 \mathrm{~s}$ before and $5 \mathrm{~s}$ after each epoch. We calculated the spike frequency using a 200-ms time window. We used a baseline from -4.5 to -4 $s$ before the first press in the sequences. We compared the spike frequency in each bin of time against the baseline, using a sliding window of $10 \mathrm{~ms}$. To resolve statistically whether the area under the curve (AUROC) was significant, we ran 1000 permutations and divided the sum of AUROC values that fall in either $>0.5$ or $<0.5$, by the number of permutations. The AUROC value was significant if the outcome was $p<0.05$. Furthermore, in each epoch, we obtained a binary matrix comparing each bin to baseline. This binary matrix was used to find the number of units modulated in each time bin. For the linear regression, to resolve statistically whether the regression's $p$ value was significant, we ran 1000 permutations and divided the sum of times that the $p$ value $>p$ value initial between the number of permutations.

\section{Stereotaxic opsin injection and fiber implantation}

For surgeries, animals were anesthetized using a mix of oxygen $(1 \mathrm{l} / \mathrm{min})$ and $1 \%$ isofluorane $(1-2 \%$ for interventional procedures). For the optogenetic experiments: after anesthesia, each animal was bilaterally injected using glass pipettes with $500 \mathrm{nl}$ of viral stock solution [either rAAV5-EF1a-DIO-eArch3.0-EYFP (Vector core, University of North Carolina), or AAV1.EF1a.DIO.eYFP.WPRE; AAV1. EF1a.DIO.hChR2(H134R)-eYFP.WPRE titer $>1 \times 1012$ (Vector core UPENN University Pennsylvania catalog \#AV-127056)] by pressure into either the M2 or M1 or lateral striatum (LS), coordinates from bregma, M2: AP 

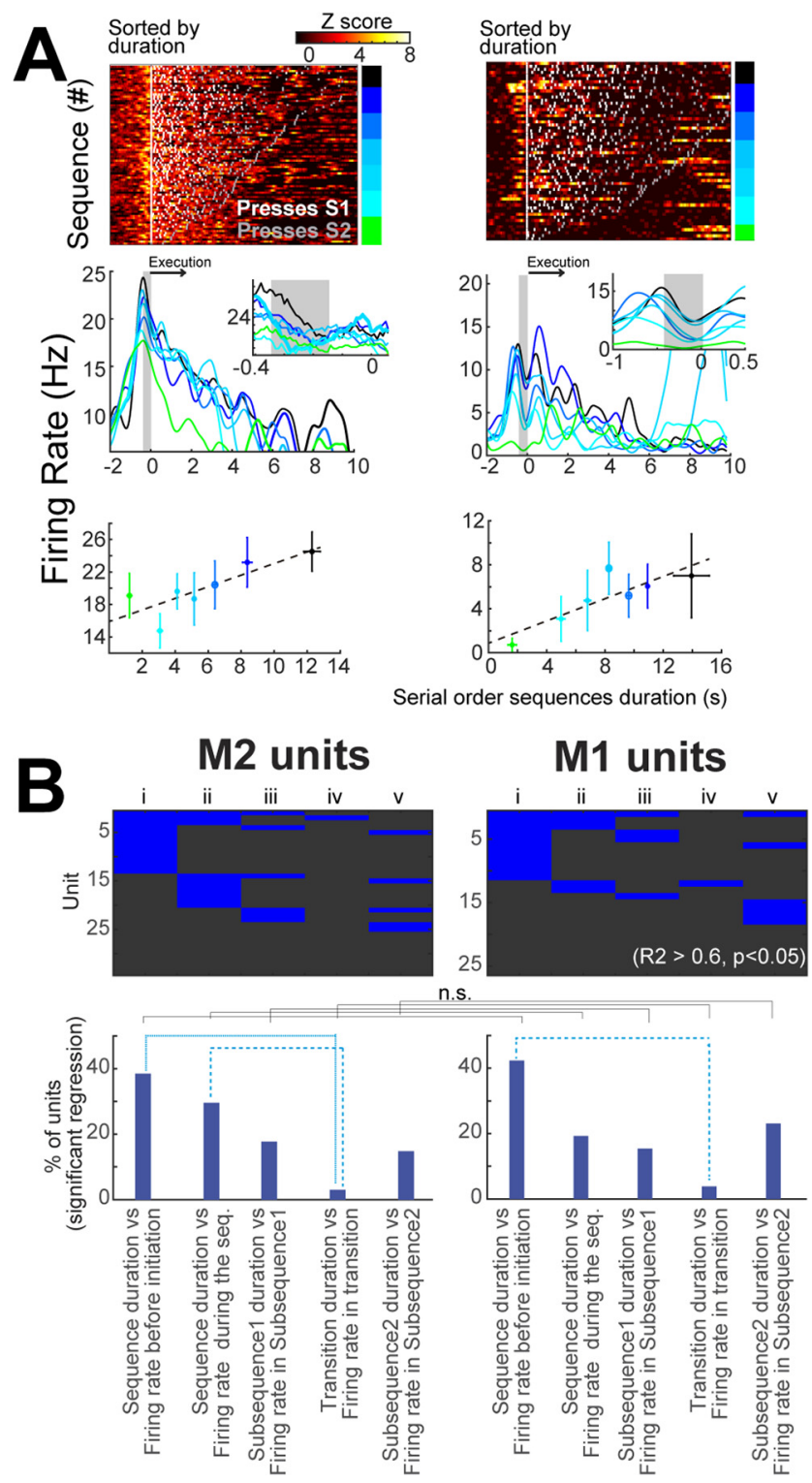

n.s.

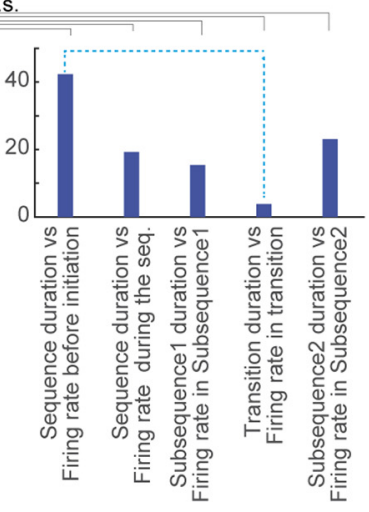

Figure 3. Both M2 and M1 contain units encoding the temporality of the self-paced sequences of actions. $\boldsymbol{A}$, top panels, Examples of two units recorded in $\mathrm{M} 2$. In each example, each line is a self-paced sequence depicting the neuronal activity (in $z$ score, black to yellow) and lever press (white points subsequence 1 , gray points subsequence 2). The color bar to the right of each plot shows the grouped categories plotted in the middle and bottom panels. Middle row panels, Mean firing rate from each category presented in the upper row. Bottom row, Regression fits from the time bin depicted in gray in the middle row panels. $\boldsymbol{B}$, top row panels, Significant regression analysis per unit; columns $\mathrm{i}$, between the firing rate (FR) $1 \mathrm{~s}$ before the start of the sequences (Seq) versus the duration of the sequences; columns ii, FR during the sequences versus duration of sequences; columns iii, FR in the subsequence 1 (S1) versus the duration of S1; columns vi, FR during the transition versus the transition time; and columns v, FR during the subsequence 2 (S2) versus the S2 duration. Bottom panels, The proportion of units that presented significant regression $\left(R^{2}>0.6\right.$ and $p<0.05)$. The dashed lines depict comparisons with $\chi^{2}$ test. Corrections for multiple comparison was considered (see Materials and Methods). Extended Data Figure 3-1 shows the continued

recorded units in M2 or M1 encoding the temporality of the forced sequences of actions. Extended Data Figure 3-2 shows the confirmation of the M2-M1 projections into the LS and linear regressions between the activity and the temporal parameters of the execution of sequences from the photo-identified M2 or M1 cortico-striatal neurons. n.s. $=p>0.05$.

$2.34 \mathrm{~mm}, \mathrm{ML} 1.25 \mathrm{~mm}$, DV 0.60-0.70 mm. M1: AP $0.5 \mathrm{~mm}$, ML $1.60 \mathrm{~mm}$, DV 0.60-0.70 mm. LS: AP $0.50 \mathrm{~mm}$, ML 2.50 $\mathrm{mm}$, DV $2.40 \mathrm{~mm}$ below the brain's surface. After the injections were done $(23 \mathrm{nl}$ every $5 \mathrm{~s}$; Nanoject II, Drummond Scientific), we waited $15 \mathrm{~min}$ to allow time for virus to spread, and a fiber-optic (300 $\mu \mathrm{m} ; \mathrm{NA} 0.37)$ was implanted into each hemisphere of the striatum. The optical fibers were fixed to the skull using acrylic cement (Lang Dental Manufacturing Co, Inc).

\section{Retrobead injections}

For the retrograde labeling experiments, $300 \mathrm{nl}$ of retrobeads (Lumafluor) were injected into the LS. Coronal sections $(50 \mu \mathrm{m})$ were obtained to determine the total number of cells labeled in the M2 or M1. The quantification was done in one slice every $300 \mu \mathrm{m}$ covering these regions.

\section{Retro-Cre injections}

Similarly, to the retrobead injections, $300 \mathrm{nl}$ of mCherryRetro-Cre (Addgene catalog \#55632-AAVrg, RRID: Addgene 55632) AAV were injected into the LS, and $300 \mathrm{nl}$ of DIO-eYFP were injected into the M2 cortex of Emx1-Cre. Coronal striatal sections of $50 \mu \mathrm{m}$ were obtained to determine the axons crossing by the dorsomedial or the dorsolateral striatum.

\section{Cortico-striatal fibers quantification}

The axonal quantification protocol was as previously reported (Díaz-Hernández et al., 2018). In short, we extracted the brains and sectioned the striatum $(50-\mu \mathrm{m}$ coronal sections). $Z$ stacks at $63 \times$ magnification were acquired (192 × $192 \times 10 \mu \mathrm{m} ; 1-\mu \mathrm{m}$ interslice) from a random quadrant; using a randomly positioned grid covering either the dorsolateral or the dorsomedial striatum (ZEN lite software, Zeiss, LSM 710). These Z stacks were imported into ImageJ; then, a maximum projection image was used to apply a filter (Hessian filter), allowing the quantification of fibers as defined by the number of fibers crossing a randomly generated line spaced $\sim 20 \mu \mathrm{m}$.

\section{Cortical microstimulation of forelimb region in the M1}

Micro-stimulation experiments were performed to identify the M1's coordinates corresponding to the contralateral forelimb region. The animal was placed in the stereotaxic apparatus under anesthesia (ketamine $0.15 \mathrm{mg} / \mathrm{g}$ mouse + xylazine $0.01 \mathrm{mg} / \mathrm{g}$ mouse). Access to motor cortex was achieved by trepanning a window of at least $1 \mathrm{~mm}$ in diameter around the center point (AP $+1, M L 1)$. Electrical stimulation was performed using a $300-\mu \mathrm{m}$ concentric bipolar electrode on the dura's surface with 15 square pulses of $200 \mu \mathrm{s}$ at a frequency of $200 \mathrm{~Hz}$ using a stimulator device 
(DS2, Digimiter). With the administration of voltage pulses, we looked for contralateral forepaw movement and corroborated this using a camera. This stimulation was performed on a grid every $100 \mu \mathrm{m}$ in the AP and ML direction. The coordinates where the stimulation resulted in movement of the contralateral brachial biceps were taken to implant the recording electrode (mouse1: AP 0.5, ML 1.5; mouse 2, AP $0.0, M L 1.5)$.

\section{Temporally defined optogenetic striatal inhibition in vivo}

Light was delivered via 300 micrometer-diameter implantable fibers (Doric lenses) coupled to a single longitudinal mode laser (MSL-FN-556, CNI lasers; $556 \mathrm{~nm}$ ). For the optogenetic inhibition experiments, a free launching system controlled by an AOM (AAoptoelectronics) and fast speed shutter (Thorlabs catalog \#SH5, SC10) triggered by TTL output from the MED-PC behavioral box was used to deliver the light. Power at the fiber tip was verified for every experiment using a power meter (Thorlabs catalog \#PM130D). The power was adjusted to be $20 \mathrm{~mW}$ at the tip of the fiber for the green light. To define the time point for the optogenetic inhibition of the cortico-striatal projections before the initiation of sequences, we took advantage of the fact that animals developed stereotypical sequences. Thus, when the animal moved from the magazine to the first lever (left lever), the infrared beam was broken, sending the timestamp to trigger the light on and allowing the quantification of the latency to initiate the sequences of actions (Figs. 1B,C, 4G; Extended Data Fig. 4-1C,I,O). To define the time point for the light manipulations during the sequences' execution, we used the timestamp of the first lever press in the sequences (Fig. 5A). To define the time point for the light manipulations during the transition between forced subsequences, we used the timestamp of the penultimate lever press of subsequence 1 . In self-paced sequences, we quantified the mean of lever presses to define a penultimate lever press (although we confirmed that it was two presses before the last of $S 1$ in this case; see Fig. 6B). During the session of optogenetic inhibition, there were control (light off) trials and stimulation trials. The stimulation trials were randomly presented throughout the session ( $50 \%$ of total trials).

\section{Behavioral quantification during optogenetic inhibitions}

The percentage of correct sequences of actions was quantified. A correct sequence was defined as the sequence with at least four presses on the first lever followed by at least four lever presses on the second lever. We also calculated the proportion of incorrect sequences (errors; Fig. 1G), by dividing the number of incorrect sequences by the total number of stimulation trials (on trials) or control light trials (off trials). Latency to initiate was calculated as the time between crossing the infrared beam out of the magazine and the first lever press in the sequences. The duration of a sequence was the time from the first press of subsequence 1 to the last press of subsequence 2 . The transition between sequences was the time from the last press in subsequence 1 to the first press in subsequence 2. All animals were video recorded during the optogenetic manipulations. This allowed us to verify that all animals used both forepaws to execute the presses.

\section{Ex vivo whole-cell recordings}

To express ChR2 in the M2 cortico-striatal projections, we injected 300 nanoliters of ChR2 into M2 ( $n=4$ mice). After 8-16d, the animals were deeply anesthetized and transcardially perfused to obtain striatal slices as described in Díaz-Hernández et al. (2018). Postsynaptic currents in whole-cell configuration were evoked by pulses of blue light (1 ms), with a pair of cells (one dMSN and one iMSN) recorded per slice. The recordings were acquired as described in Díaz-Hernández et al. (2018).

\section{Experimental design and statistical analysis}

Significance was determined by $p<0.05$. For the proportions, paired or between groups, the $\chi^{2}$ test, the Wilcoxon test, or the Mann-Whitney $U$ test was used, as appropriate. For comparisons along sessions, a non-parametric Friedman statistical test was used. When multiple comparisons were employed, a Benjamini-Hochberg correction was used to adjust the $p$ value (Fig. 3B; Extended Data Figs. 3-1A, 3-2G). All statistical analyses were performed using GraphPad, R [R core Team (2013)] and MATLAB. Additionally, for the optogenetic manipulations experiments, we use estimation statistics based on confidence intervals (Cls) as described in Ho et al. (2019) and Manouze et al. (2019). The effect sizes and Cls are reported as: effect size [ $\mathrm{Cl}$ width lower bound; upper bound]. Five thousand bootstrap samples were taken, and the $p$ value(s) reported are the likelihood(s) of observing the effect size(s), if the null hypothesis of zero difference is true. To account for multiple comparisons at each inhibition protocol, we considered a false discovery rate (FDR)-adjusted $p$ value ( $q$ value) $<0.10$, two-sided, as significant (Storey, 2002).

\section{Data and software availability}

All data and MATLAB scripts will be available on request to the lead or corresponding authors.

\section{Results}

To identify the premotor cortico-striatal contribution to serial order sequence execution, we developed a behavioral task in mice allowing us to perform two types of experiments. Mice were trained to execute two subsequences of lever presses on two levers in serial order (Fig. 1). For the first experiment, we aimed to identify whether neuronal activity in M2 or M1 is modulated during the task's serial order sequences. We performed in vivo electrophysiological recordings in $\mathrm{M} 2$ or $\mathrm{M} 1$ to measure neuronal activity while animals executed these lever press sequences (Figs. 2, 3). For the second experiment, we aimed to identify whether the projections from these cortical regions contribute to the sequences' execution, by optogenetically inhibiting the corticostriatal projections either before or during the sequences' execution, or during the transition between the two subsequences (Figs. 4-6). 
A
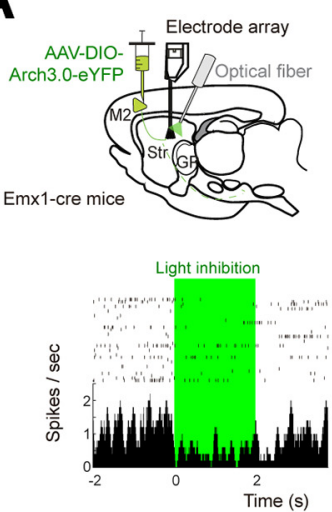

B

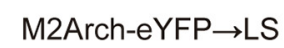
$(\mathrm{M} 2 \rightarrow \mathrm{LS})$
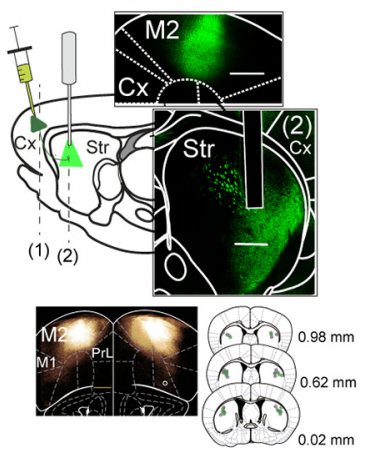
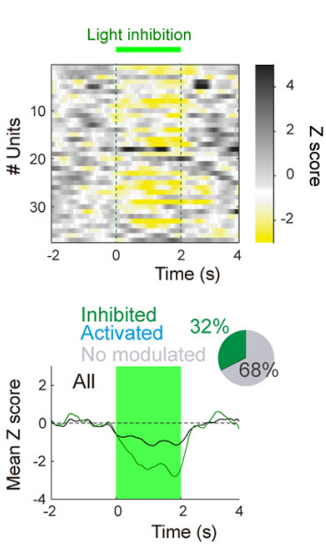

M1Arch-eYFP $\rightarrow$ LS
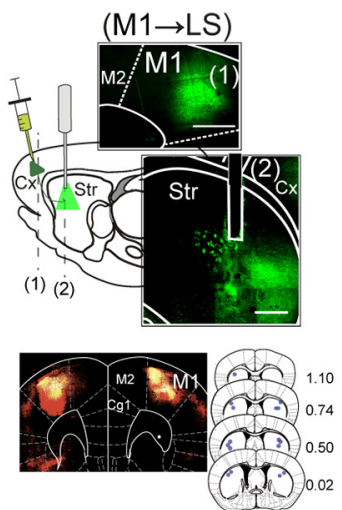

C
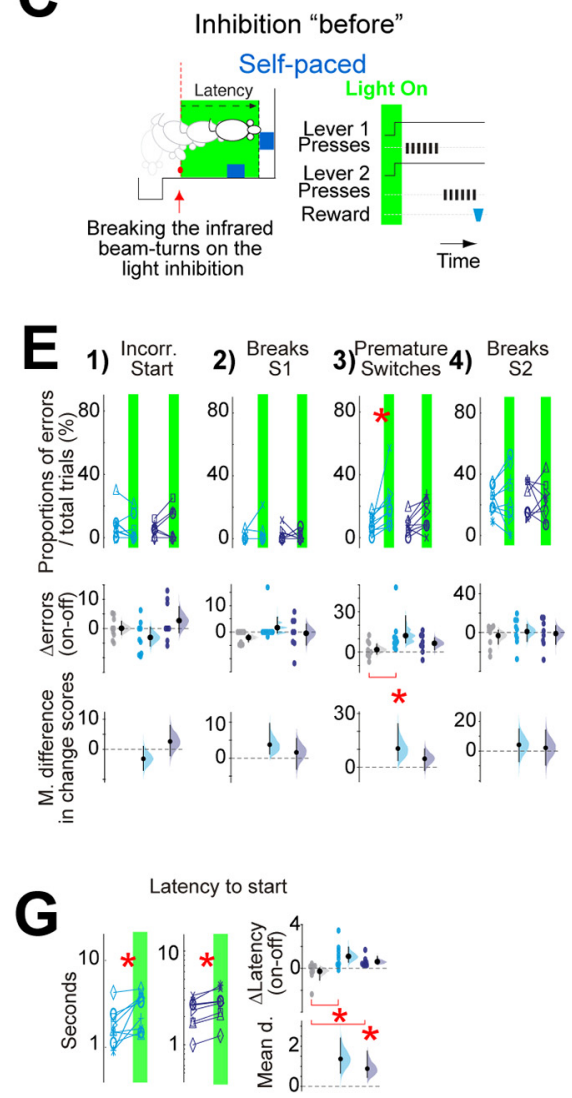

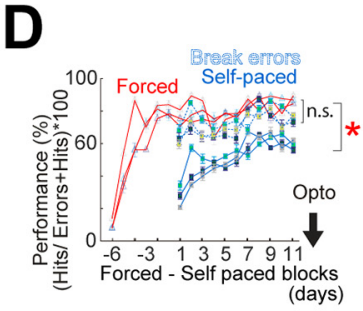

F

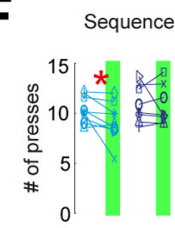

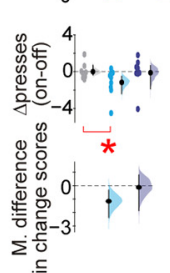

M2 $\rightarrow$ LS

M1 $\rightarrow$ LS

Control

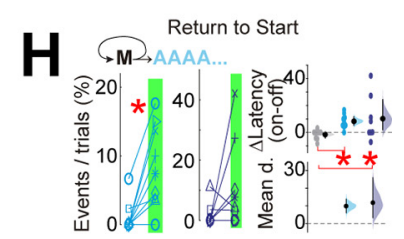

Figure 4. The inhibition of the premotor and primary motor cortico-striatal projections before sequence initiation impairs the initiation, but only the premotor disrupts the execution. $\boldsymbol{A}$, top left, Diagram illustrates the injection site of Arch3.0-eYFP and the optrode (electrode array + fiber optic) implantation into the LS. Top right, Plot depicting the activity of several units recorded in the striatum ( $z$ score) aligned to the inhibition of the M2 cortico-striatal projections into the LS (green line above). Bottom left, representative perievent time histogram and raster plot of a striatal unit's activity aligned to the inhibition of the M2 cortico-striatal projections (green shadow). Bottom right, Mean z score for the units that decreased their activity (green) or for all units recorded (black) during the inhibition. Inset, Pie chart showing the proportion of modulated and non-modulated units (comparing baseline time vs light). $\boldsymbol{B}$, top panels, Sagittal diagrams and cortical photomicrographs of the injection of Arch3.0-eYFP into either M2 $(n=9)$ or M1 $(n=8)$ cortices and optical fibers implantation into the LS (Str). Scale bar: $500 \mu \mathrm{m}$. Bottom panels, Photomicrograph showing the average of Arch3.0-eYFP expression of the corresponding groups. Coronal diagrams representing the position of the optical fiber's tips into the LS (green dots: Arch3.0-eYFP; gray dots: eYFP). C, Scheme of the inhibition protocol before the initiation of the sequences. The light inhibition ( $2 \mathrm{~s}$ ) is triggered by breaking the infrared beam positioned outside of the magazine, when coming to the lever (dashed red lines on the schemes). D, Percentage of correct sequences [correct/(errors + correct)] throughout training. $\boldsymbol{E}$, upper panels 1-4, Quantification per animal in off versus on trials of the proportion of each category of error. $\boldsymbol{F}$, Effect of the inhibition on the number of lever press per sequence. $\mathbf{G}$, Effect of the inhibition of the cortico-striatal projections on the latency to initiate self-paced sequences. $\boldsymbol{H}$, Effect of the inhibition of the cortico-striatal projections on the proportions of times per block that animals returned to the magazine after having crossed the infrared sensor outside of the magazine (which set the timestamp to trigger the light inhibition; see Movie 3). On the paired plots, each line depicts the mean effect per animal during trials of optogenetic inhibition (on; green shadow) versus trials without optogenetic inhibition (off; no shadow) from the same session. In panels $\boldsymbol{E}-\boldsymbol{H}, \boldsymbol{\Delta}$ on-off panels are obtained from the mean difference per animal in the on-off trials, adding the control group in gray. The mean difference in change score panels (mean d.) are obtained between the experimental groups and the control group (see Materials and Methods). Paired plots, two-sided permutation $t$ test; panels $\Delta$ on-off, Arch animals versus control eYFP animals, unpaired two-sided permutation $t$ test; ${ }^{*} p<0.05$. The exact $p$ values are described in the text and Table 1. Extended Data Figure $4-1$ shows the effects of the inhibition of the lateral striatal neurons during the initiation and execution of the action sequences. Extended Data Figure 4-2 shows the M2/M1 retrogradely labeled cells from the LS, the thalamus, and the pons. Extended Data Figure 4-3 shows that the premotor cortico-striatal projections innervate the direct pathway with a stronger synaptic weight than the indirect pathway. n.s. $=p>0.05$.

\section{Mice can learn to execute serial order sequences of lever presses}

To investigate the contribution of cortico-striatal synapses to the structuring and execution of self-paced serial order sequences, we trained mice in a serial order task to execute two subsequences of lever presses (Fig. 1A). The execution of a correct serial order sequence was achieved when animals performed at least four presses on a lever 1 (subsequence 1; S1) followed by four presses on lever 2 (subsequence 2; S2), which lead to the delivery of a 
A

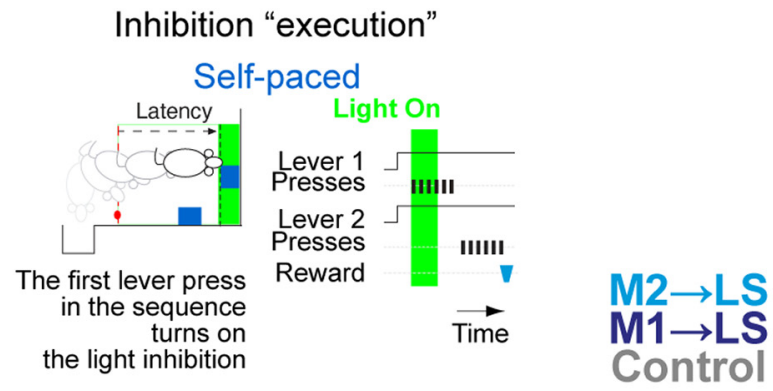

B

1) Incorr.

2) Breaks

3) $\begin{aligned} & \text { Premature } \\ & \text { Switches 4) Breaks }\end{aligned}$

C

Sequence
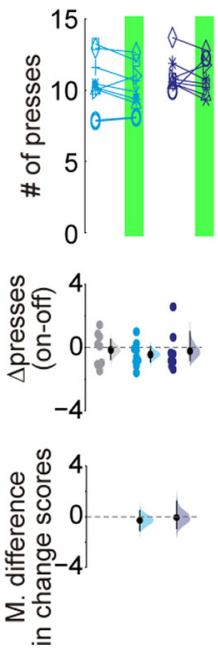

D

Long S1 sequences
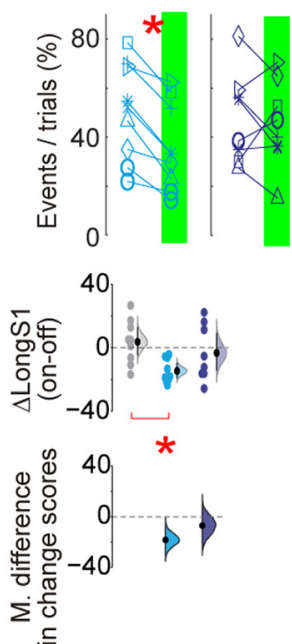

Figure 5. The inhibition of premotor cortico-striatal projections at the start of the execution decreased Long-S1 self-paced sequences. $\boldsymbol{A}$, Scheme of the inhibition execution protocol. Light inhibition was triggered by the first press in the sequences. The green shadow depicts $2 \mathrm{~s}$ of continuous light inhibition. $\boldsymbol{B}$, upper panels 1-4, Quantification per animal in off versus on trials of the proportion of each category of error. $\boldsymbol{C}$, Effect of the inhibition on the number of lever presses per sequence. $\boldsymbol{D}$, As in $\boldsymbol{B}, \boldsymbol{C}$ evaluating the proportion of animals performing Long-S1 sequences $\mathrm{S} 1>4 \rightarrow \mathrm{S} 2>=4$ ). On the paired plots, each line depicts the mean effect per animal during trials of optogenetic inhibition (on; green shadow) versus trials without optogenetic inhibition (off; no shadow) from the same session. In panels $\boldsymbol{B}-\boldsymbol{D}, \boldsymbol{\Delta}$ on-off panels are obtained from the mean difference per animal in the on-off trials, adding the control group in gray. The mean difference in change score panels (mean d.) are obtained between the experimental groups and the control group (see Materials and Methods). Paired plots, two-sided permutation $t$ test; panels $\Delta$ on-off, Arch animals versus control eYFP animals, unpaired two-sided permutation $t$ test; ${ }^{*} p<0.05$. The exact $p$ values are described in the text and Table 1 .

reward in the magazine (pellet). To execute self-paced sequences, we first trained animals to execute forced sequences (forced: only one of the two levers was exposed at any point, signaling the animals when to press; Fig. 1B). Later in training, blocks of forced and self-paced serial order sequences were intercalated (self-paced: both levers remained exposed so that animals decided when to press and transition between subsequences; Fig. 1C). The requirement of intercalated blocks was necessary as animals' performance dropped when they were required to perform only self-paced sequences (tested in a group of eight animals: data not shown). The main difference between a forced and a self-paced serial order sequence was that in the former, completing four presses on lever 1 retracted it and exposed lever 2; if four presses were executed on lever 2, it was also retracted, followed by the delivery of a pellet in the magazine (Movie 1). On the other hand, the execution of a correct self-paced sequence also required at least four presses on each lever, but in this case, both levers remained exposed throughout the self-paced trials (Movie 2).

A session containing both different kinds of sequences is identified in the figures as blocks of "forced-selfpaced" sessions. During one of these sessions, the animals were required to achieve correct blocks of five forced and five self-paced sequences consecutively for $30 \mathrm{~min}$. Figure $1 D$ shows an example animal during the first (early) and the eleventh (late) day (rewards are labeled with filled red triangles for forced and in blue for self-paced). After $21 \mathrm{~d}$ of training, and $9 \mathrm{~d}$ performing blocks forced-self-paced sessions, animals showed a stable performance of correct sequences, demonstrated by the fact that performance no longer changed from sessions 9 to 11 [performance forced, $\chi^{2}(2)=2.51, p=0.30$, performance self-paced, $\chi^{2}(2)=1.0$, 
A

Inhibition "transition"
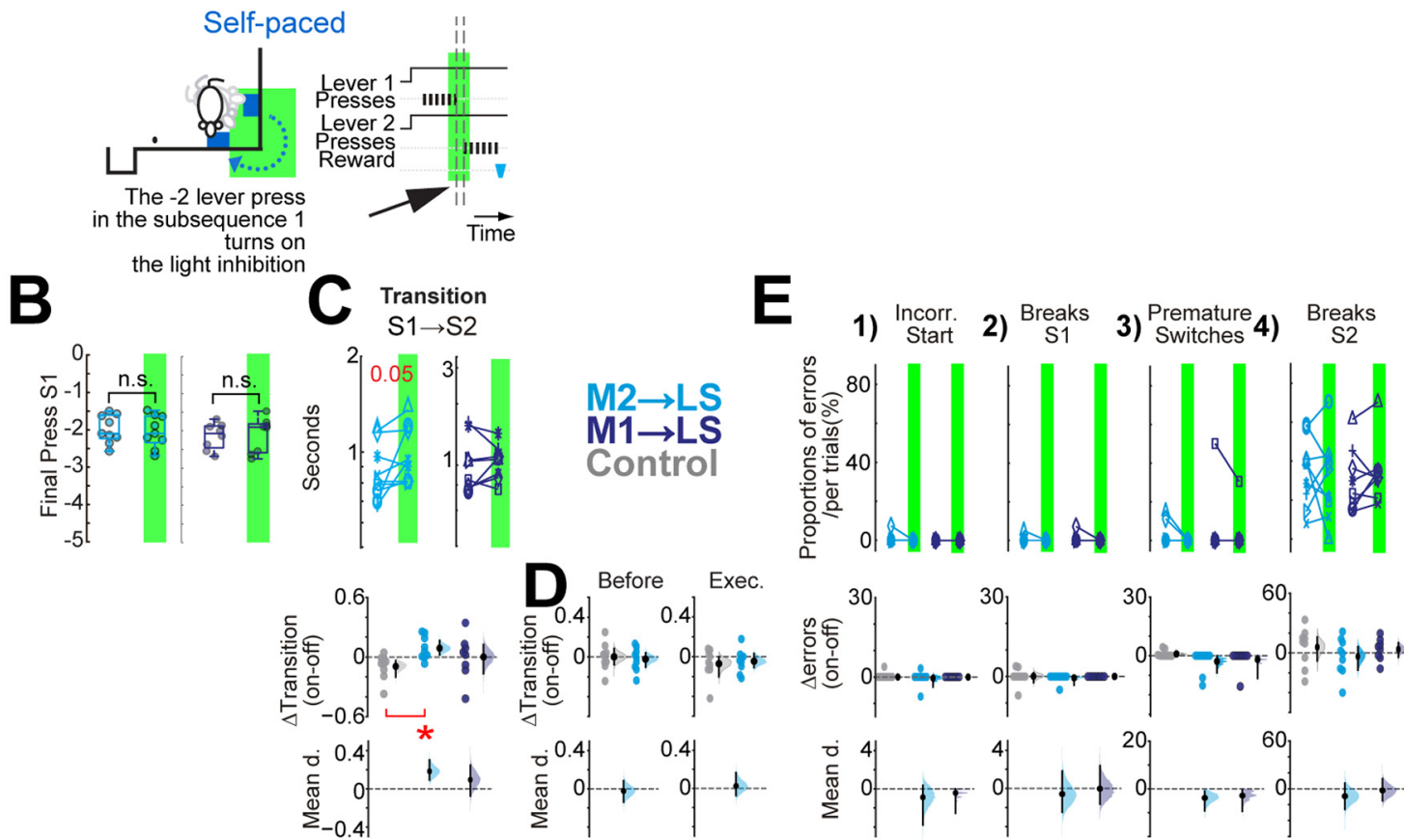

Figure 6. Inhibition of premotor but not primary motor cortico-striatal projections at the moment of the transition increases the transition time inside self-paced sequences. $\boldsymbol{A}$, Scheme of the inhibition protocol transition in self-paced sequences. The light inhibition was triggered by the press before the penultimate press of the subsequence 1 . The dashed and black arrows denote the period of the transition. $\boldsymbol{B}$, Estimation of the press that delivered the timestamp for light inhibition for M2 $\rightarrow$ LS and M1 $\rightarrow$ LS, light and dark blue, respectively. $\boldsymbol{C}$, upper panels, Effect of the inhibition of the premotor or primary motor cortico-striatal projection on the transition time for self-paced sequences. $\boldsymbol{D}, \Delta$ on-off for the inhibition before and execution protocols from the inhibition of M2 $\rightarrow$ LS presented in Figures 4, 5, respectively. $\boldsymbol{E}$, No effect was detected by the inhibition of either M2 $\rightarrow$ LS or M1 $\rightarrow$ LS projection in the different categories of errors. Upper panels 1-4, Quantification per animal in off versus on trials of the proportion of each category of error. In $\boldsymbol{C}, \boldsymbol{E}$, each line in the paired plots depicts the mean effect per animal during trials of optogenetic inhibition (on; green shadow) versus trials without optogenetic inhibition (off; no shadow) from the same session. The panels $\Delta$ on-off are obtained from the mean difference per animal in the on-off trials, adding the control group in gray. The mean difference in change score panels (mean d.) are obtained between the experimental groups and the control group (see Materials and Methods). Paired plots, twosided permutation $t$ test; panels $\Delta$ on-off, Arch animals versus control eYFP animals, unpaired two-sided permutation $t$ test; ${ }^{*} p<0.05$. The exact $p$ values are described in the text and Table 1.

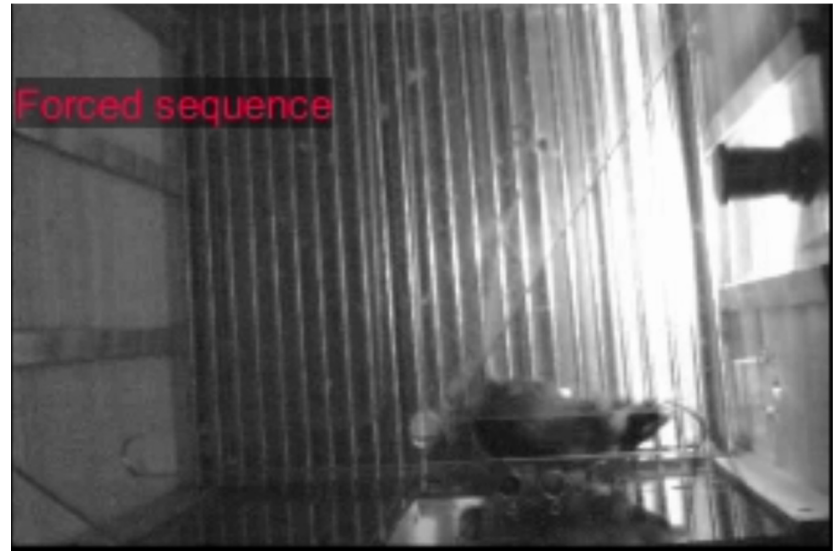

Movie 1. Execution of a forced sequence. [View online]

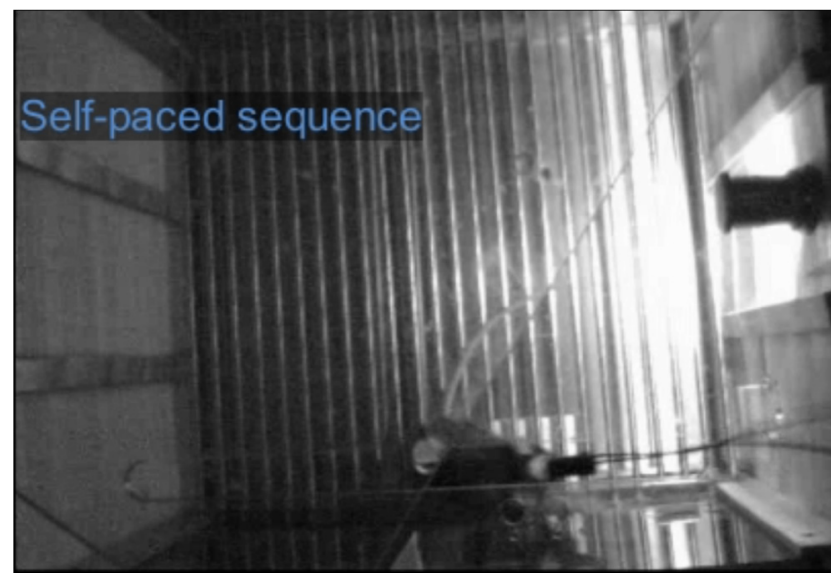

Movie 2. Execution of a self-paced sequence. [View online] 


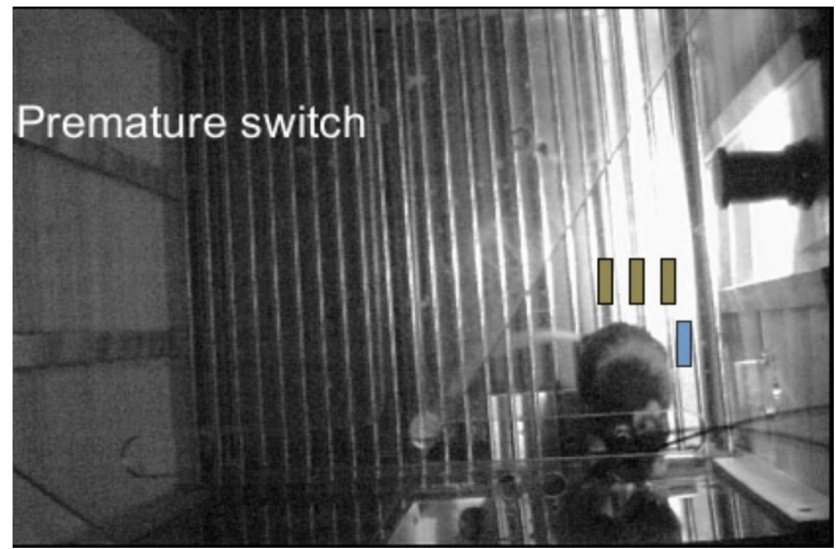

Movie 3. Example of a broken sequence after light inhibition before the initiation of the sequence. [View online]

$p=0.65$, Friedman test, wild-type (WT) animals, $n=8$, Fig. $1 E$. No differences were found in comparisons of a number of task-relevant parameters between forced versus selfpaced sequences: latency to initiate, the transitions intrasubsequences, the intervals between presses intra subsequences $(p>0.05$, Mann-Whitney $U$ test, WT animals, $n=8$; Extended Data Fig. 1-1A).

While animals executing forced sequences reached an $83 \pm 1.5 \%$ in correct performance (correct sequences/errors + correct sequences), the execution of self-paced sequences only reached $61 \pm 1.7 \%$ (WT animals, $n=8$; Fig. $1 E$, red and blue tick curves, respectively). To further investigate this performance, we quantified the types of errors and modes in which the animals executed correct sequences. An error was defined as a sequence of presses in which the animal did not achieve four presses on lever 1 followed by four presses on lever 2 . The errors were grouped into four categories: (1) incorrect start (starting on the opposite lever); (2) breaks in S1; (3) premature switches from S1 to S2; and (4) breaks in S2 (Fig. 1B,C, right panels $1-4$, respectively; note that 1 and three were only possible during self-paced). These four categories were quantified late in training (Fig. 1G). The decrease in Breaks in S1 and S2 was the primary determinant in performance improvement of both forced (ForcBreaks) and self-paced (S-PBreaks) sequences compared with pretraining $(p<0.05$, MannWhitney $U$ test, WT animals, $n=8$; Extended Data Fig. $1-1 B$, panels 2, 4). A decrease in incorrect-start errors accounted for the major improvement in the self-paced performance $(p=0.007$, Mann-Whitney $U$ test, WT animals, $n=8$; Extended Data Fig. 1-1B, panel 1). No difference in performance between forced and self-paced sequences was detected when accounting for only the available errors in both forced and self-paced sequences: BreaksS1+BreaksS2 $(p=0.5$, Mann-Whitney $U$ test, WT animals, $n=8$; Fig. $1 E$, blue dashed curve vs red curve).

The correct execution in forced sequences was $\mathrm{S} 1=4 \rightarrow \mathrm{S} 2=4$; in self-paced the execution of correct sequences grouped in several options (as anything with $\mathrm{S} 1=$ $>4 \rightarrow \mathrm{S} 2>=4$ was correct). The majority of correct

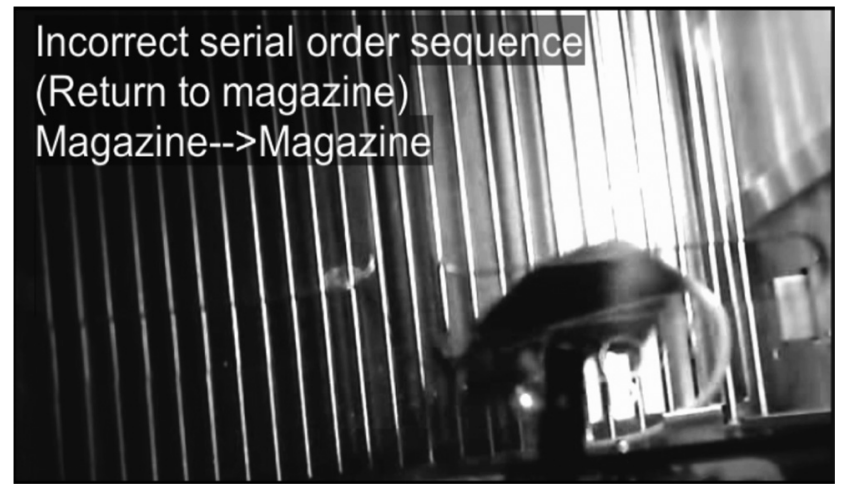

Movie 4. Example of return to start after light inhibition before the initiation of the sequence. [View online]

sequences were Long-S1 sequences $(S 1>4 \rightarrow S 2>=4$; WT animals, $n=8$; Fig. $1 F$ ).

Besides the execution structure of the sequences, we quantified the length parameters. The latency to start, the transition between subsequences, the interpress intervals, the duration, all became faster as training progressed (Friedman test, $p<0.05$; WT animals, $n=8$; Extended Data Fig. 1-1A). Later in training, the latency to initiate the sequences was not different between forced and self-paced (forced: $1.1 \pm 0.1 \mathrm{~s}$, self-paced: $1.5 \pm 0.3$ $\mathrm{s}, p=0.535$, Mann-Whitney $U$ test, WT animals, $n=8$; Fig. $1 H)$. Neither the transition time between subsequences $\left(\mathrm{S} 1_{\text {end }} \rightarrow \mathrm{S} 2_{\text {start }}\right)$ or the interpress intervals intersubsequences between the two modes $(p>0.05$, Mann-Whitney $U$ test; Fig. $1 \mathrm{~J}, K)$. However, the duration and the number of presses were longer in the $\mathrm{S} 1$ of self-paced than in forced [forced duration: $3.2 \pm 0.2 \mathrm{~s}$ vs self-paced duration $_{\text {in }}: 4.8 \pm 0.4 \mathrm{~s}$, forced $_{\text {s1 presses }}=4 \pm 0$ vs $6.1 \pm 0.6$ in self-paced s1 presses, $_{\text {, }}$ $p=0.0002$, Mann-Whitney $U$ test; Extended Data Fig. 1$1 C, D)$.

This task parameterization showed that mice could learn and execute forced and self-paced serial order sequences in blocks. The main difference in the execution of these two modes of sequences (besides that self-paced have more possibilities for errors) is that animals execute longer chunks of presses in the subsequence 1 of selfpaced sequences.

\section{Activity modulation in premotor and motor cortical neurons during the execution of serial order sequences}

Once we established that mice are capable of executing serial order sequences, we questioned if, as predicted, the activity of M2 and M1 cortical neurons was modulated during the execution of these trained sequences (Tanji and Evarts, 1976; Mushiake et al., 1990; Churchland et al., 2006; Shima et al., 2007). For this purpose, we trained eight animals as in Figure 1. After $3 \mathrm{~d}$ in the blocks of forced-self-paced sessions, we performed brain surgeries to implant a mobile electrode array either in $\mathrm{M} 2$ ( $n=6$; Fig. $2 A$, middle panel) or M1 $(n=2$; here, we verified the region to evoke forepaw movements by micro- 
stimulation; see Materials and Methods; Fig. $2 A$, right panel). After $4 \mathrm{~d}$ of recovery, the training restarted until animals reached a stable performance in the execution of forced and self-paced sequences (10-15 blocks sessions postsurgery). From these animals, 34 well-isolated units in M2 and 26 units in M1 were analyzed. Representative examples of these neurons' activity during the executions of sequences are presented in Figure 2B,C. The $z$ score heatmaps of Figure 2D,E show all recorded units during the execution of self-paced sequences (the same units during the execution of forced sequences are presented in Extended Data Fig. 2-1).

To answer whether the same units were active during the different phases of the sequences, we measured the mean activity of neurons above two $z$ scores during the execution of the sequences. We classified neuronal activity into categories based on different parameters of sequence execution (Fig. 2F). We found no statistical difference in the comparison of M2 versus M1 activity, only a tendency for more modulated/engaged M2 neurons related to the initiation of a sequence (Fig. 2G). To explore this difference during sequence initiation, we compared the mean $z$ score between M2 and M1 activity from the units that showed a significant modulation $1 \mathrm{~s}$ before starting the sequences (Fig. $2 \mathrm{H}, I)$. This comparison showed a bigger $z$ score positive modulation in M2 than in M1 during the execution of the sequences (M2 $=14$ of 34 units, $M 1=8$ of 26 units; mean $z$ score in self-paced sequences: $\mathrm{M}_{\mathrm{S} 1}=1.32, \mathrm{M}_{\mathrm{S} 2}=0.85$ vs $\mathrm{M} 1_{\mathrm{s} 1}=0.55, \mathrm{M} 1_{\mathrm{s} 2}=$ $0.22 ; p<0.05$, $z$ score difference test; Fig. $2 \mathrm{~J}$, bars labeled "S1 and S2"). Remarkably M2 units showed a stronger modulation in self-paced sequences, even before the initiation of the sequence $\left(\mathrm{M}_{\mathrm{S}_{\text {S-Pinit }}}=1.4 \mathrm{vs} \mathrm{M} 1_{\mathrm{S} \text {-Pinit }}=0.8\right.$, $p=0.04 z$ score difference test; Fig. $2 \mathrm{~J}$, bars labeled "Init" in blue) and a similar tendency during the initiation of forced sequences (Extended Data Fig. 2-1H, bars labeled "Init" in red). No difference in the number of recruited units throughout the execution of the sequences was detected ( $p>0.05, \chi^{2}$ test; Extended Data Fig. 2-1/).

\section{Both M2 and M1 contain units encoding the length of the serial order sequences of actions}

After the evaluation of the mean $z$ score related to the execution of the serial order sequences, we tested the hypothesis that M2/M1 encode the length parameters of the execution of these sequences (Riehle and Requin, 1989; Riehle et al., 1994; Lucchetti and Bon, 2001; Sakurai et al., 2004; Churchland et al., 2006; Jin et al., 2009; Hernández et al., 2010; Merchant et al., 2013; Crowe et al., 2014; Murakami et al., 2014; Mendoza et al., 2018). To evaluate this possibility, we performed linear regressions between the duration of the segments of the sequences (total sequence, S1, transition, or S2) and the neural activity recorded from individual neurons in either M2 or M1. We reasoned that if these cortices contained activity encoding the sequence length, their cortico-striatal projections could convey this information to the striatum.

Figure $3 A$ shows two examples of units that presented significant regressions $\left(R^{2}>0.6\right.$ and $\left.p<0.05\right)$ in specific time bins during the execution of serial order self-paced sequences (Fig. $3 A$, middle row panels, gray shadows). Figure $3 B$ shows the proportion of units that presented with significant regressions based on five questions: column i, does the activity before the first press $(1 \mathrm{~s})$ encode the sequences' length? ii, does the mean activity during the sequence encode the length of the sequence? iii, iv, and $\mathrm{v}$, Is there a relationship between the duration of $\mathrm{S} 1$, the transition or the duration of $\mathrm{S} 2$, and the mean firing rate during these epochs? The answer to these five questions is presented per recorded unit in either M2 or M1 (Fig. 3B, upper panels) and as the $\%$ of units with significant regressions per region (Fig. $3 B$, bottom panels). Notably, the biggest proportion of units with significative regressions was between the sequence duration and the firing rate before the initiation of the sequence in both $\mathrm{M} 2$ and M1 (Fig. 3B, columns i) or the sequences duration and the mean firing rate of $\mathrm{M} 2$ during the sequences (Fig. $3 B$, columns ii).

The percentage of recruited units over time (units presenting significant regressions, $200-\mathrm{ms}$ bin, sliding $10 \mathrm{~ms}$; $R^{2}>0.6$ and $p<0.05$ ) is presented in Extended Data Figure 3-1B. Despite small tendencies, no difference was detected in the proportion of units recruited overtime either when comparing $\mathrm{M} 2$ versus $\mathrm{M} 1$ nor when comparing within each region between forced and self-paced sequences $(1 \mathrm{~s}$ before or one after the start of the sequences). Similarly, no differences were found between neuronal activity and the duration of the sequence, duration of $\mathrm{S} 1$, duration of the transition, or the number of presses (comparison of bars $p>0.05, \chi^{2}$ test; Extended Data Fig. 3-1B).

To address whether specific cortico-striatal M2 or M1 neurons encode for sequence parameters, we recorded from antidromic photo-identified cortico-striatal neurons in vivo (Lima et al., 2009; Díaz-Hernández et al., 2018). To achieve this, we first confirmed the lateral region of the striatum (LS) that is innervated by both M2 and M1 projections (Extended Data Fig. 3-2A-D; Oh et al., 2014; Sohur et al., 2014; Hintiryan et al., 2016; Hunnicutt et al., 2016). Then we trained mice in which the expression of Channelrhodopsin-2 (ChR2) was targeted to the excitatory cortical neurons in M2 $(n=4)$ or M1 $(n=2)$ and their projections to the striatum (using Emx1-Cre mice; Guo et al., 2000; Gorski et al., 2002). We then implanted an electrode array above the ChR2 expression and an optical fiber into LS to antidromically activate the cortico-striatal units (Extended Data Fig. 3-2E-G; Friedman et al., 2015). Following this procedure and after a stable performance in the execution of blocks of forced-self-paced sequences, the activity of M2 or M1 units was recorded. During these recordings, we recorded cortical and cortico-striatal photo-identified units (PID units). A PID unit was that, at the end of the session, responded to antidromic light stimulation with short latency ( $<10 \mathrm{~ms}$; Díaz-Hernández et al., 2018). The mean latency of $\mathrm{M} 2+\mathrm{M} 1 \rightarrow \mathrm{LS}$ was $5.1 \pm 0.4 \mathrm{~ms} ; n=21$; Extended Data Fig. $3-2 F$, bottom right panel). Following these criteria, we identified $10 \mathrm{M} 2$ and $11 \mathrm{M} 1$ cortico-striatal PID units (Extended Data Fig. $3-2 G$ ). As in the non-PID units (Fig. 3B), the major proportion of PID units presented significant regressions between the sequences duration and the firing rate before 
the initiation, the duration, and the mean firing rate during the execution of the sequences (Extended Data Fig. 3-2G, columns i, ii).

In summary, in Figures 2, 3 and Extended Data Figure $3-2$, we show that the activity of M2 and M1 is related to the execution of the trained sequences. The mean $z$ score analysis showed that $\mathrm{M} 2$ had a bigger modulation than M1 during the execution and even before the initiation of the serial order sequences. Furthermore, the regression analysis showed that both M2 and M1 contain units that encode the sequences' execution length.

\section{Inhibition of lateral striatal neurons before initiation of a serial order sequences impairs its execution}

This study's main goal was to establish whether cortico-striatal projections of the premotor and motor cortex contribute to the execution of sequences. To address this point, we first verified that neuronal activity in the lateral striatal (LS) region that receives inputs from M2 and M1 (Extended Data Fig. 3-2A-D) contributes to the execution of serial order sequences. For this purpose, we trained a group of animals in which the inhibitory opsin archaerhodopsin (Arch3.0) was injected bilaterally into LS followed by fiberoptic implantation above the injection sites (Extended Data Fig. 4-1A). Then, once the animals reached around $80 \%$ success (correct sequences) in the performance of forced and $60 \%$ in self-paced sequences (Extended Data Fig. 4-1A, panel 3), we performed sessions of optogenetic inhibition, delivering the light to inhibit Arch3.0-expressing neurons in a state-dependent manner during the execution of the sequences. We randomized three protocols (each protocol on a different day): (1) before the initiation; (2) during the execution; or (3) during the transition between subsequences (Extended Data Fig. 4-1B-G, $H-M, N-S$, respectively). We observed that optogenetic inhibition before starting the sequence delayed the initiation (Extended Data Fig. 4-1C). Inhibition during the execution of the sequence increased premature switches (Extended Data Fig. 4-1J, panel 3) and decreased the correct Long-S1 self-paced sequences (Extended Data Fig. 4-1K). Inhibition during the intersubsequence transitions increased the transition time between forced subsequences and showed a tendency to increase the time between self-paced subsequences (Extended Data Fig. 4-1R). Note that the data for the forced sequences are only presented in the figures when significant effects were detected; otherwise, they are presented only in Table 1.

\section{Presequence initiation inhibition of either motor or premotor cortico-striatal projections impairs initiation, but only the latter disrupts the execution}

Once we verified that direct inhibition of LS neurons impaired the execution of sequences, we asked whether the premotor $(\mathrm{M} 2 \rightarrow \mathrm{LS})$ or the motor $(\mathrm{M} 1 \rightarrow \mathrm{LS})$ cortico-striatal projections contributed to the execution of these sequences. We first verified the inhibition of striatal neurons by the optogenetic inhibition of cortico-striatal projections using Arch3.0 with 2-s pulses of light (Fig. $4 A$ ). This pulse length is in a proper range far from the biophysical constraints of using Arch for optogenetic inhibition (Mahn et al., 2016;
Díaz-Hernández et al., 2018). Furthermore, we evaluated the proportion of labeled cells in M2/M1 that contain corticofugal axons crossing by the area of the LS manipulations (Extended Data Fig. 4-2).

Next, we tested whether the contribution of corticostriatal projections is time dependent (Bartolo et al., 2014; Bartolo and Merchant, 2015; Li et al., 2016; Nakayama et al., 2018). We performed optogenetic inhibitions of the $\mathrm{M} 2 \rightarrow \mathrm{LS}(n=9)$ or M1 $\rightarrow \mathrm{LS}(n=8)$ projections in a state-dependent manner, using three protocols: (1) before initiation (Fig. 4); (2) during execution (Fig. 5); and (3) during the transition between subsequences (Fig. 6).

Most of the effects of optogenetic inhibition were on self-paced sequences. Therefore, for Figures 4-6, forced sequences are only presented when effects were detected; otherwise, only self-paced sequences are presented. The full data for forced and self-paced inhibitions is in the Table 1.

To carry out these experiments, a group of Emx1-Cre mice was subject to bilateral Arch3.0-eYFP expression into M2 or M1 (or eYFP for control animals) and bilateral fiber optic implantation into the LS (Fig. 4B; see Figs. 4-6). After surgery, animals were allowed to recover for $3 \mathrm{~d}$ before training started and continued until the performance was stable (Fig. 4D). During the optogenetic inhibition sessions, and depending on the protocol, a continuous pulse of green light was applied randomly in $50 \%$ of the trials ( $2 \mathrm{~s}$; triggered by the behavior), allowing us to compare the effects of light inhibition on each animal within the same session.

For the inhibition "before" initiation protocol, we aimed to assess whether the cortico-striatal projections contribute to sequence execution by interfering with the initiation/preparation of the sequences for which we took advantage of the stereotyped behavior of the trained animals. An infrared beam was placed between the magazine and the levers (red arrow-dashed line coming out of the magazine; Fig. $4 C$ ), it was possible to trigger a pulse of light when the animal crossed the infrared beam before starting the sequences (Tecuapetla et al., 2016).

The inhibition of the $\mathrm{M} 2 \rightarrow$ LS projections before initiation increased premature switches (M2 $\rightarrow \mathrm{LS}_{\text {Self-paced }} \mathrm{On}=20 \pm 5$ vs $\mathrm{M} 2 \rightarrow \mathrm{LS}_{\text {Self-paced }}$ off $=8 \pm 1$; the paired mean difference was $12.2[95.0 \% \mathrm{Cl} 6.8,27]$ and $p=0.0001, q=0.01$ twosided permutation $t$ test; Fig. $4 E$, panel 3 , light blue data; Movie 3). This effect was not observed in control animals ( $\Delta$ on-off comparison to the control group: the unpaired mean difference between control and $\mathrm{M} 2 \rightarrow$ LS was $10.4[95.0 \% \mathrm{Cl}$ $3.7,2.4]$. The $p$ value of the two-sided permutation $t$ test was 0.0224, $q=0.06$; Fig. $4 F$, panel 3, bottom part). The increased premature switches were accompanied by decreased presses within the self-paced sequence $\left(\mathrm{M} 2 \rightarrow \mathrm{LS}_{\text {Self-paced }} \mathrm{On}=9 \pm 0.6\right.$ vs $\mathrm{M} 2 \rightarrow \mathrm{LS}_{\text {Self-paced }} \mathrm{Off}=$ $10 \pm 0.4$; the paired mean difference was $-1.16[95.0 \%$ $\mathrm{Cl}-2.42,-0.495], p=0.016, q=0.05$ two-sided permutation $t$ test; Fig. $4 F$, light blue data).

This inhibition protocol also increased the latency to start the sequences $\left(\mathrm{M} 2 \rightarrow \mathrm{LS}_{\text {Self-paced_Latency }}\right.$ on $=2.9 \pm 0.4$ vs $\mathrm{M} 2 \rightarrow \mathrm{LS}_{\text {Self-paced_Latency }} \mathrm{Off}=1.8 \pm 0.3$; the paired mean difference was $1.1[95.0 \% \mathrm{Cl} 0.556,1.96], p=0.0026$, $q=0.01$ two-sided permutation $t$ test; Fig. $4 G$, light blue 
left panel; forced sequences were also delayed, see Table 1, data related to Fig. 4), an effect not observed in control animals (Fig. 4G, $\Delta$ on-off panel, gray data). This increased latency to start was accompanied by an increase in the animals' return to the magazine in the trials that received light inhibition, suggesting that inhibition interrupted the proper serial order sequence initiation (see Movie 4; M2 $\rightarrow$ LS SP-returns to starton $=$ $9 \pm 2 \%$ vs $\mathrm{M} 2 \rightarrow \mathrm{LS}_{\mathrm{SP} \text {-returns_to_start }} \mathrm{off}=1 \pm 0.7 \%$, the paired mean difference between control and $\mathrm{M} 2 \rightarrow \mathrm{LS}$ was $8.29[95.0 \% \mathrm{Cl} 5.14,11.7], p=0.006, q=0.03$ twosided permutation $t$ test. Unpaired mean difference between control and $\mathrm{M} 2 \rightarrow$ LS was $9.86[95.0 \% \mathrm{Cl} 6.0$, 13.8], $p=0.0002, q=0.002$ two-sided permutation $t$ test; Fig. 4G, $\Delta$ on-off panel).

Conversely to the inhibition of $\mathrm{M} 2 \rightarrow$ LS projections, the increase in premature switches and the decrease in the number of presses within the sequence were not observed when we inhibited $\mathrm{M} 1 \rightarrow$ LS projections (Fig. $4 E, F$, dark blue data). Instead the inhibition of the $\mathrm{M} 1 \rightarrow$ LS projections increased the latency to start the self-paced sequences $\left(\mathrm{M} 1 \rightarrow \mathrm{LS}_{\text {Self-paced_Latency }} \mathrm{On}=\right.$ $3.2 \pm 0.4$ vs $\mathrm{M} 1 \rightarrow \mathrm{LS}_{\text {Self-paced Latency }} \mathrm{Off}=2.6 \pm 0.3$; the paired mean difference was $0.614[95.0 \% \mathrm{Cl} 0.40,1.1]$ and $p=0.009, q=0.04$ of the two-sided permutation $t$ test; Fig. $4 G$, comparison to control: panel $\Delta$ on-off same figure, dark blue vs gray data, unpaired mean difference between control and $\mathrm{M} 1 \rightarrow$ LS was $0.88[95.0 \%$ $\mathrm{Cl} 0.4,1.7]$ and $p=0.003, q=0.01$ of the two-sided permutation $t$ test), and the returns to start (comparison to control: panel $\Delta$ on-off in the same figure, dark blue vs gray data; unpaired mean difference between control and $\mathrm{M} 1 \rightarrow \mathrm{LS}$ was $11.8[95.0 \% \mathrm{Cl} 3.3,26.1]$ and $p=0.02$, $q=0.06$ of the two-sided permutation $t$ test; Fig. $4 H$ ).

Together these results suggest that the $\mathrm{M} 2 \rightarrow$ LS but not the $\mathrm{M} 1 \rightarrow \mathrm{LS}$ projections contribute to the execution/structuring of self-paced sequences while both $\mathrm{M} 2 \rightarrow$ LS and $\mathrm{M} 1 \rightarrow$ LS contribute to the initiation.

\section{Inhibition of premotor cortico-striatal projections at the start of the execution decreased Long-S1 sequences}

One prediction for the cortico-striatal projections' contribution to serial order sequences execution is that their requirement would be time dependent (see Figs. 2, 3; Li et al., 2016; Nakayama et al., 2018). To further prove this idea, we performed a second protocol: inhibition during "execution," which consisted of performing light inhibition of the cortico-striatal projections once the execution of the sequences started (triggered by the first press in $\mathrm{S} 1$; Fig. 5A). Using this protocol, we observed that the inhibition of the M2 $\rightarrow$ LS projections did not modify the proportion of the different categories of errors (Fig. 5B) nor the number of presses in the sequences (Fig. $5 C$ ), but it did decrease the proportion of Long-S1 sequences $\left(\mathrm{M} 2 \rightarrow \mathrm{LS}_{\text {Long_S1_Seq }} \mathrm{On}=35 \pm 5\right.$ vs $\mathrm{M} 2 \rightarrow$ LS Long_S1_Seqoff $=$ $50 \pm 6$, the paired mean difference was $-14.6[95.0 \% \mathrm{Cl}$ $-18.9,-9.8]$ and $p=0.002, q=0.04$ of the two-sided permutation $t$ test; Fig. $5 D$, upper panels; comparison to control eYFP-group: $\Delta$ on-off panel, unpaired mean difference between control and $\mathrm{M} 2 \rightarrow$ LS was $-18.4[95.0 \% \mathrm{Cl}-28.3$, $-8.8] p=0.003, q=0.04$ of the two-sided permutation $t$ test Fig. $5 D$, bottom panel), with no overall effect on the duration or the number of presses in the sequences (see Table 1; data related to Fig. 5). The inhibition of the $\mathrm{M} 1 \rightarrow$ LS projections did not yield any significant effects with the inhibition during execution protocol (Fig. 5, dark blue data).

The results from the inhibition of $\mathrm{M} 2 \rightarrow \mathrm{LS}$ or $\mathrm{M} 1 \rightarrow \mathrm{LS}$ projections during the beginning of the sequence execution highlight that the activity of the $\mathrm{M} 2 \rightarrow$ LS projections is important for the length of the sequences (particularly for S1). Furthermore, these results also suggest that, at least later in training, the $\mathrm{M} 1 \rightarrow$ LS projections are not required for sequence execution.

\section{Inhibition of premotor cortico-striatal projections during the transition between serial order subsequences increases the transition time}

So far, we have shown that the cortico-striatal projections from $\mathrm{M} 2 \rightarrow$ LS contribute to the appropriate initiation and execution of self-paced serial order sequences (Figs. $4,5)$. However, in the previous experiments, the light inhibition never occurred during the transition between subsequences. Given that a small proportion of units from either M2 or M1 showed significant modulation during the transition-moment (Fig. 2F,G), we asked whether inhibiting directly during the transition could reveal whether the $\mathrm{M} 2 \rightarrow \mathrm{LS}$ or $\mathrm{M} 1 \rightarrow \mathrm{LS}$ contribute to the transition. For this purpose, we set up an inhibition during "transition" proto$\mathrm{col}$, in which the press before the penultimate press of $\mathrm{S} 1$ triggered light inhibition for $2 \mathrm{~s}$ (Fig. 6A, for self-paced; in forced sequences, it was the third press; see Table 1, data related to Fig. 6).

Figure $6 B$ shows the mean press in which the animals of the $\mathrm{M} 2 \rightarrow \mathrm{LS}$ or the $\mathrm{M} 1 \rightarrow \mathrm{LS}$ groups received the inhibitory stimulation (or the corresponding timestamp in the "off" trials); on average, it was the press -2 counting from the final press in the $\mathrm{S} 1 \quad(\mathrm{M} 2 \rightarrow \mathrm{LS}$ transition light $\mathrm{On}=-2.0 \pm 0.1 \mathrm{vs}$ $\mathrm{M} 2 \rightarrow \mathrm{LS}_{\text {transition light }}$ off $=-1.9 \pm 0.1, p=0.25$, Mann-Whitney $U$ test; Fig. 6B). With this protocol, we observed that the inhibition of $\mathrm{M} 2 \rightarrow \mathrm{LS}$, but not $\mathrm{M} 1 \rightarrow \mathrm{LS}$, showed a tendency to increase the duration of the transition $\left(\mathrm{M} 2 \rightarrow \mathrm{LS}_{\text {Transition }} \mathrm{On}=\right.$ $1.0 \pm 0.06$ vs $\mathrm{M} 2 \rightarrow \mathrm{LS}_{\text {Transition }}$ off $=0.8 \pm 0.06$, the paired mean difference was $0.08[95.0 \% \mathrm{Cl} 0.02,0.16]$ and $p=0.050, q=0.4$ of the two-sided permutation $t$ test; Fig. $6 C$, upper panels), that reached significance when compared with the eYFP-control group: unpaired mean difference between control and $\mathrm{M} 2 \rightarrow \mathrm{LS}$ was $0.18[95.0 \% \mathrm{Cl}-0.09,0.3]$ and $p=0.003, q=0.03$ of the two-sided permutation $t$ test; Fig. $6 C, \Delta$ on-off bottom panel). This effect was time-specific since neither the inhibition before nor the inhibition execution protocol on the M2 $\rightarrow$ LS yielded a similar effect (Fig. $6 D$ ). This effect on the transition raised the question of whether the M2 $\rightarrow$ LS projections treat each subsequence as independent chunks or concatenate them into a single chunk once the whole sequence has been acquired (Geddes et al., 2018; Martiros et al., 2018). We hypothesized that if the transition was affected by the light inhibition, the proportion of breaks in the sequences might increase as well. Contrary to this hypothesis, no modifications on the breakings of sequences 
were detected by this protocol (Fig. 6E). We also observed no differences in the number of presses or sequence length (Table 1), supporting the idea that the $\mathrm{M} 2 \rightarrow \mathrm{LS}$ projections contribute to the transition intersubsequences without affecting the second part of the serial order sequence.

Altogether, the results from the recording and inhibition experiments reveal that the cortico-striatal projections to the LS from the premotor and motor cortices have specific contributions to the execution of sequences. Both projections contribute to the initiation, while $\mathrm{M} 2 \rightarrow \mathrm{LS}$ also contributes to the correct execution and transition between subsequences of self-paced sequences. Importantly the $\mathrm{M} 2 \rightarrow$ LS contribution was essential during the beginning of the execution, suggesting its contribution is relevant before the initiation, at the beginning of execution, and decreasing in relevance as the sequence execution progressed.

\section{Premotor cortico-striatal projections innervate the direct pathway with a stronger synaptic weight than the indirect pathway}

Finally, we investigated whether the M2 $\rightarrow$ LS projections differentially impact the two subcircuits of striatal projection neurons, the direct or indirect striatal pathways (Wall et al., 2013), by looking at synaptic connectivity. For these experiments, we crossbred Emx1-Cre (allowing us to express ChR2 in M2) with BAC D2-GFP mice. By injecting red retrobeads into the substantia nigra, we could identify in the striatum neurons from direct pathway (red retrobeads; dMSN), or the indirect pathway (GFP; iMSN). This allowed us to record from identified striatal neurons in brain slices ex vivo while stimulating the $\mathrm{M} 2 \rightarrow$ LS ChR2 axons (Extended Data Fig. 4-3). Using this procedure, we recorded pairs of neurons in the same brain slice in the LS (one iMSN and one dMSN; counterbalancing the recording order per slice). We evoked postsynaptic responses by activating the M2 $\rightarrow$ LS ChR2 projections with brief pulses of light $(1 \mathrm{~ms}$; Extended Data Fig. 4-3C). From these experiments, we recorded a total of 6 pairs of striatal neurons (in 6 different slices from four animals). Postsynaptic evoked responses had a bigger amplitude in the dMSN than in the iMSN (dMSN: $290 \pm 100$ vs iMSN $=30 \pm 9$ pA, MannWhitney $U$ test, $p=0.031$; Extended Data Fig. 4-3F), with no difference in their latencies (dMSN: $3.3 \pm 0.1 \mathrm{~ms}$ vs $\mathrm{iMSN}=3.8 \pm 0.1 \mathrm{~ms}$, Mann-Whitney $U$ test, $p>0.05$; Extended Data Fig. 4-3F). These evoked postsynaptic responses were glutamatergic, being blocked by the AMPA receptor antagonist CNQX (Extended Data Fig. $4-3 G)$. This finding suggests that the $M 2 \rightarrow$ LS projections may have stronger synaptic connections onto the dMSNs than onto the iMSNs in vivo when the animals are executing the serial order action sequences.

\section{Discussion}

These results show that mice can execute serial order forced and self-paced sequences of lever presses (Fig. 1). Both the premotor (M2) and motor (M1) cortices are modulated during sequence execution (Fig. 2). M2 showed a bigger modulation during the initiation and execution in the units modulated before the start of the sequences (Fig. 2J). Both M2 and M1 units showed regressions between their activity and the sequence duration before or during the execution (Fig. 3B), even in photo-identified M2 and M1 corticostriatal cells (Extended Data Fig. 3-2G). The M2 $\rightarrow$ LS and M1 $\rightarrow$ LS projections contribute to the proper initiation, but mainly $\mathrm{M} 2 \rightarrow$ LS projections contribute to the structuring/execution of serial order self-paced sequences (Figs. 4-6). The M2 $\rightarrow$ LS synapses present bigger amplitude postsynaptic responses onto the direct versus the indirect pathway of striatal neurons (Extended Data Fig. 4-3).

Mice had been previously been shown to develop and execute serial order operant tasks, including pressing two levers (Geddes et al., 2018; Rothwell et al., 2015). The task adapted here allows temporally separated manipulations within the different phases of the two subsequences (initiation, execution, and transition between subsequences). We first trained animals in forced sequences and later in blocks of forced-self-paced sequences as animals trained only in the latter dropped their behavior. The fact that animals were more efficient at executing forced than self-paced can be explained by the greater number of possible errors in self-paced sequences. However, a difference in the recruitment of brain structures in forced versus self-paced sequences is not excluded (Romo and Schultz, 1992; Hernández et al., 2010).

To identify whether the cortico-striatal projections from M2/M1 contribute to sequence execution, we investigated whether neuronal activity in these structures was modulated during the execution of the sequences. We identified that M2 and M1 units showed activity modulations milliseconds before the start and during the execution of these serial order sequences (Figs. 2, 3), with mostly exclusive firing categories (Fig. $2 F$ ). Importantly, the units from M2 that were modulated before the sequences started showed a bigger positive modulation during the execution and before starting the sequences than those from M1 (Fig. 2J). This increased activity time before the execution of movements is attributed to time estimation or preparation to execute an action [e.g., the anticipatory activity (ramping) in $\mathrm{M} 2$ has a direct relationship with the time that animals wait to start an action or the duration of a stimulus (Murakami et al., 2014; Mendoza et al., 2018)]. In this study, the animals had to approach the lever before the first press in the sequence. We could not therefore rule out that the activity before starting the sequences may be related to motor preparation, although the inhibition of this activity before pressing delayed the latency of both forced and self-paced sequences, but only impaired the execution of selfpaced sequences. Together, these results show that units in M2/M1 are modulated during the execution of sequences. When evaluating whether these units' activity may encode the execution parameters, we observed no significant differences in the proportions of units in $M 2$ versus $M 1$, as measured by regression analysis between neuronal activity and temporal parameters of the sequences (Fig. $3 B$ ). These results show that both M2 and $\mathrm{M} 1$ contain units encoding the sequences' execution (Nelson et al., 2020; Berlot et al., 2021). However, it must be acknowledged that the lack of differences in some of the comparisons between M2 and M1 may be 
influenced by the small set of recorded cells (34 cells from $\mathrm{M} 2$ and 26 from M1).

To evaluate how the M2/M1 cortico-striatal projections contribute to the execution of serial order sequences, we performed two experiments. First, we recorded from corticostriatal antidromic PID units (Extended Data Fig. 3-2). Second, we performed state-dependent temporal inhibitions of the $\mathrm{M} 2 \rightarrow \mathrm{LS}$ or the $\mathrm{M} 1 \rightarrow \mathrm{LS}$ projections either before or during the execution of sequences (Figs. 4-6). Although the photo-identification of cortico-striatal $\mathrm{M} 2 \rightarrow \mathrm{LS}$ or $\mathrm{M} 1 \rightarrow \mathrm{LS}$ was low (10 per structure), it allowed us to detect units in each of these cortices that presented significant regressions with temporal parameters of the execution of the sequences. However, there were too few photo-identified neurons to infer whether M2 or M1 has a more important contribution to sequences execution. The contribution of these cortico-striatal projections was thus mainly evaluated with state-dependent optogenetic inhibition.

To test the hypothesis that the M2/M1 cortico-striatal projections have time-specific contributions to sequences execution, we performed three protocols of inhibition while animals performed lever pressing sequences. Our inhibitions were all $2 \mathrm{~s}$, below the safe limit of the biophysical constraints of using Arch for optogenetic inhibitions (Mahn et al., 2016).

Interestingly, the inhibition of either the $\mathrm{M} 2 \rightarrow \mathrm{LS}$ or $M 1 \rightarrow$ LS, before the initiation increased the latency to start the sequences (Fig. 4G). This increase in latency was accompanied by an increase in the animals' return to the magazine in the initiations that received light inhibition, as if the inhibition interrupted the proper initiation (Fig. 4H; see Movie 4). These results are consistent with a model where the $\mathrm{M} 2 \rightarrow \mathrm{LS}$ and $\mathrm{M} 1 \rightarrow \mathrm{LS}$ cortico-striatal projections are required for the proper initiation of sequences, with the former likely setting up the parameters for the upcoming sequence (for further discussion, see below).

Remarkably, the three inhibition protocols revealed that the projections from $\mathrm{M} 2 \rightarrow \mathrm{LS}$, but not the $\mathrm{M} 1 \rightarrow \mathrm{LS}$, are temporally required for the execution of self-paced sequences. The inhibition of $\mathrm{M} 2 \rightarrow$ LS before the start altered the sequence structure, increasing premature switches (Fig. $4 E$ ) and decreasing the number of actions in the sequence (Fig. 4F). Their inhibition during the beginning of the execution affected the first segment of the sequence (by decreasing the probability of executing Long-S1 sequences; Fig. 5D). Their inhibition during the transition increased the transition time between subsequences, although this last had the slightest effect (Fig. 6C).

A previous experiment, which decreased the $\mathrm{M} 2 \rightarrow$ striatal projections (by manipulating $\mathrm{M} 2 \rightarrow$ striatal projections plus their collaterals to other brain regions) during the execution of a serial order short sequence reported that inhibition of $\mathrm{M} 2 \rightarrow$ dorsolateral striatal cells, retrogradely labeled from the striatum, impaired the first step accuracy (Rothwell et al., 2015), perhaps by increasing incorrect starts or promoting premature switches from S1 to S2. Here, the possibility of longer subsequences in the sequence allowed for measuring incorrect starts, breaks, premature switches, and the transition time between subsequences. Our inhibitions of $\mathrm{M} 2 \rightarrow \mathrm{LS}$ was spatially and temporally specific (if the optogenetic inhibition does not backpropagate as it has been documented; El-Gaby et al., 2016), showed no effect on incorrect starts. Rather we observed an increase in premature switches when inhibiting before the start, a decrease in Long-S1 sequences when inhibiting during the execution, and slowness of the transition time when inhibiting around the transition.

Another important point reported here is the specificity of the effects mainly on self-paced sequences (see Table 1). How is it that the premotor projections can differentiate between the execution of forced and self-paced sequences? A possible explanation is that the $\mathrm{M} 2 \rightarrow$ LS projections could detect whether the animals were in blocks of self-paced sequences, engaging these projections with a bigger sensitivity to the inhibitions as a consequence of self-deciding the execution. This possibility is supported by previous studies showing that when animals engage in self-paced behaviors, as opposed to forced behaviors, some cortical subcircuits become engaged and more important (Romo and Schultz, 1992; Hernández et al., 2010; although see Kurata and Tanji, 1985; Thaler et al., 1988). An idea supported by the tendency of cortico-striatal M2 PID units to show a bigger proportion of units with significant regression in the self-paced sequences (Extended Data Fig. 3-2G, columns i, iii). Importantly we observed that the inhibition of the $\mathrm{M} 1 \rightarrow$ LS did not affect the execution of sequences, consistent with the idea that $\mathrm{M} 1 \rightarrow \mathrm{LS}$ activity is not required to execute sequences once these have been learned (Kawai et al., 2015).

Finally, another remarkable finding from the optogenetic inhibitions experiments is that the M2 $\rightarrow$ LS projections may contribute as a serial driver of sequence execution (Lashley, 1951; Zeigler and Gallistel, 1981; Graybiel, 1998; Rosenbaum et al., 2007; Jin and Costa, 2015). Supporting this idea, their inhibition before the starting could restart the initiation, increasing premature switches during the execution, and decreasing the number of actions (Fig. 4E,F). Conversely, their inhibition during the start of the execution decreased the probability of executing Long-S1 sequences only (Fig. 5D).

In addition to the inhibition of cortico-striatal projections, we verified the LS requirement for the sequence execution and addressed the possibility that our corticostriatal inhibitions may affect other brain areas besides the LS by performing two experiments: (1) we directly inhibited the lateral striatal neurons with the same protocols that inhibited the cortico-striatal projections (Extended Data Fig. 4-1); and (2) we evaluated whether the manipulated region contains axons en passant from M2/M1 that reach other brain areas (e.g., thalamus/pons; Th/Pns; Extended Data Fig. 4-2).

The first experiment's results found that direct inhibition of LS during sequence execution decreased the length of correct sequences (Long-S1 sequence) and the number of presses (Extended Data Fig. 4-1K,M). The inhibition of the LS in the transition protocol increased the transition time between subsequences (Extended Data Fig. 4-1R). These effects were in line with the inhibition of $\mathrm{M} 2 \rightarrow$ LS projections. However, the LS inhibition before the sequence start did not change the errors in self-paced sequences, contrary to the inhibition of the $\mathrm{M} 2 \rightarrow$ LS projections which increased premature switches with this protocol. A possible explanation for 
Table 1: Descriptive statistics and data extended from the main figures

\begin{tabular}{|c|c|c|c|c|c|}
\hline & Panel/data & Descriptive statistics & Type of test & $p$ value & $n$ \\
\hline Data related to & Breaks S1 & Mean \pm SEM (\%) & & & \\
\hline \multirow[t]{30}{*}{ Figure 4} & & Forced off vs forced on & & & \\
\hline & & M2-LS: $0 \pm 0$ vs $1.8 \pm 0.9 ;$ M1-LS: $0.8 \pm 0.8$ vs $2.3 \pm 1.2 ;$ con- & Paired two-sided & $1 ; 1 ; 1$ & Emx1-cre mice $(+/-)$ \\
\hline & & trols: $0 \pm 0$ vs $2.6 \pm 1.4$ & permutation $t$ test & & AAV-Arch-M2-LS: $n=9$ \\
\hline & & Forced: change rate (on-off) & & & AAV-Arch-M1-LS: $n=8$ \\
\hline & & M2-LS: $1.8 \pm 0.9$ vs controls: $2.6 \pm 1.4$ & Unpaired two-sided & 0.68 & Control: \\
\hline & & M1-LS: $1.4 \pm 0.8$ vs controls: $2.6 \pm 1.4$ & permutation $t$ test & 0.49 & AAV-eYFP-M2-LS: $n=9$ \\
\hline & Breaks S2 & Mean \pm SEM (\%) & & & \\
\hline & & Forced off vs forced on & & & \\
\hline & & $\begin{array}{l}\text { M2-LS: } 13.7 \pm 3.3 \text { vs } 7.8 \pm 2.9 ; \text { M1-LS: } 12 \pm 6 \text { vs } 19 \pm 7 \text {; con- } \\
\quad \text { trols: } 17 \pm 4 \text { vs } 14 \pm 3\end{array}$ & $\begin{array}{l}\text { Paired two-sided } \\
\text { permutation } t \text { test }\end{array}$ & $0.1 ; 0.3 ; 0.2$ & \\
\hline & & Forced: change rate (on-off) & & & \\
\hline & & M2-LS: $5.8 \pm 3$ vs controls: $-3 \pm 2.6$ & Unpaired two-sided & 0.84 & \\
\hline & & M1-LS: $6.9 \pm 6$ vs controls: $-3 \pm 6.9$ & permutation $t$ test & 0.79 & \\
\hline & Sequence & Mean \pm SEM (\# presses) & & & \\
\hline & & Forced off vs forced on & & & \\
\hline & & $\begin{array}{l}\text { M2-LS: } 8.3 \pm 0.1 \text { vs } 8.4 \pm 0.2 ; \text { M1-LS: } 8.1 \pm 0.2 \text { vs } 8 \pm 0.1 \text {; con- } \\
\quad \text { trols: } 8.1 \pm 0.1 \text { vs } 8 \pm 0.1\end{array}$ & $\begin{array}{l}\text { Paired two-sided } \\
\text { permutation } t \text { test }\end{array}$ & $0.8 ; 0.6 ; 0.7$ & \\
\hline & & Forced: change rate (on-off) & & & \\
\hline & & M2-LS: $0.07 \pm 0.3$ vs controls: $-0.03 \pm 0.09$ & Unpaired two-sided & 0.84 & \\
\hline & & M1-LS: $-0.08 \pm 0.1$ vs controls: $-0.03 \pm 0.09$ & permutation $t$ test & 0.79 & \\
\hline & Latency & Mean \pm SEM (s) & & & \\
\hline & & Forced off vs forced on & & & \\
\hline & & $\begin{array}{l}\text { M2-LS: } 1.4 \pm 0.1 \text { vs } 2.4 \pm 0.3 ; \text { M1-LS: } 2.9 \pm 0.6 \text { vs } 3.6 \pm 0.6 \\
\quad \text { controls: } 2.0 \pm 0.4 \text { vs } 1.8 \pm 0.3\end{array}$ & $\begin{array}{l}\text { Paired two-sided } \\
\text { permutation } t \text { test }\end{array}$ & $0.0001^{\star \star \star} ; 0.23 ; 0.3$ & \\
\hline & & Forced: change rate (on-off) & & & \\
\hline & & M2-LS: $1.0 \pm 0.2$ vs controls: $-0.2 \pm 0.2$ & Unpaired two-sided & $0.0016^{\star \star}$ & \\
\hline & & M1-LS: $0.7 \pm 0.6$ vs controls: $-0.2 \pm 0.2$ & permutation $t$ test & 0.11 & \\
\hline & Return to start & Mean \pm SEM $(\%)$ & & & \\
\hline & & Forced off vs forced on & & & \\
\hline & & $\begin{array}{l}\text { M2-LS: } 0.7 \pm 0.7 \text { vs } 6 \pm 3 \text {; M1-LS: } 2.7 \pm 1 \text { vs } 13 \pm 5 \text {; controls: } \\
\quad 1 \pm 1 \text { vs } 1 \pm 0.7\end{array}$ & $\begin{array}{l}\text { Paired two-sided } \\
\text { permutation } t \text { test }\end{array}$ & $0.1 ; 0.06 ; 0.8$ & \\
\hline & & Forced: change rate (on-off) & & & \\
\hline & & M2-LS: $5.7 \pm 2.9$ vs controls: $-0.06 \pm 1$ & Unpaired two-sided & 0.11 & \\
\hline & & M1-LS: $10.7 \pm 5.4$ vs controls: $-0.06 \pm 1.9$ & permutation $t$ test & $0.04^{*}$ & \\
\hline \multirow{23}{*}{$\begin{array}{l}\text { Data related to } \\
\text { Extended } \\
\text { Data Figure } \\
4-1\end{array}$} & Performance & Data presented in the figure & & & \\
\hline & & Forced vs self-paced & Mann-Whitney $U$ test & $0.0001^{\star \star \star}$ & C57BL6/J mice (WT) \\
\hline & & Forced vs S-P-break errors & & 0.32 & AAV-Synapsine-Arch-M2-LS: $n=8$ \\
\hline & & & & & Control group $n=16$ \\
\hline & Latency & Mean \pm SEM (s) & & & \\
\hline & Inhibition & Forced off vs forced on & & & \\
\hline & before & $\begin{array}{l}\text { LS: } 2.5 \pm 0.2 \text { vs } 4.2 \pm 0.6 ; \text { controls: } 2.5 \pm 0.3 \text { vs } 2.1 \pm 0.33 \\
\text { Forced: change rate (on-off) }\end{array}$ & $\begin{array}{l}\text { Paired two-sided } \\
\text { permutation } t \text { test }\end{array}$ & $0.02^{\star} ; 0.3$ & \\
\hline & & LS: $1.6 \pm 0.8$ vs controls: $-0.3 \pm 0.2$ & Mann-Whitney $U$ test & $0.003^{\star \star}$ & \\
\hline & Breaks S1 & Mean \pm SEM $(\%)$ & & & \\
\hline & Inhibition & Forced off vs forced on & Paired two-sided & & \\
\hline & before & LS: $7.4 \pm 4$ vs $9 \pm 4$; controls: $0.8 \pm 0.8$ vs $1.8 \pm 0.8$ & permutation $t$ test & $0.38 ; 0.55$ & \\
\hline & & Forced: change rate (on-off) & Unpaired two-sided & & \\
\hline & & LS: $2 \pm 2$ vs controls: $0.9 \pm 1.3$ & permutation $t$ test & 0.67 & \\
\hline & Breaks S2 & Mean \pm SEM (\%) & & & \\
\hline & Inhibition & Forced off vs forced on & & & \\
\hline & before & $\begin{array}{l}\text { LS: } 20 \pm 6.2 \text { vs } 11 \pm 3.7 ; \text { controls: } 17.7 \pm 3.4 \text { vs } 14.9 \pm 2.4 \\
\text { Forced: change rate (on-off) }\end{array}$ & $\begin{array}{l}\text { Paired two-sided } \\
\text { permutation } t \text { test }\end{array}$ & $0.06 ; 0.29$ & \\
\hline & & LS: $-8.7 \pm 3$ vs controls: $-2 \pm 2.5$ & $\begin{array}{l}\text { Unpaired two-sided } \\
\text { permutation } t \text { test }\end{array}$ & 0.20 & \\
\hline & Long-S1 se- & Mean \pm SEM (\%) & & & \\
\hline & quences & Forced off vs forced on & Paired two-sided & $1.0 ; 0.51$ & \\
\hline & Inhibition & LS: $0 \pm 0$ vs $1.1 \pm 1$; controls: $1.8 \pm 1$ vs $0.9 \pm 0.5$ & permutation $t$ test & & \\
\hline & before & Forced: change rate (on-off) & Unpaired two-sided & 0.36 & \\
\hline & & LS: $1.1 \pm 1$ vs controls: $-0.8 \pm 1.1$ & permutation $t$ test & & \\
\hline & & (Continued) & & & \\
\hline
\end{tabular}


Table 1: Continued

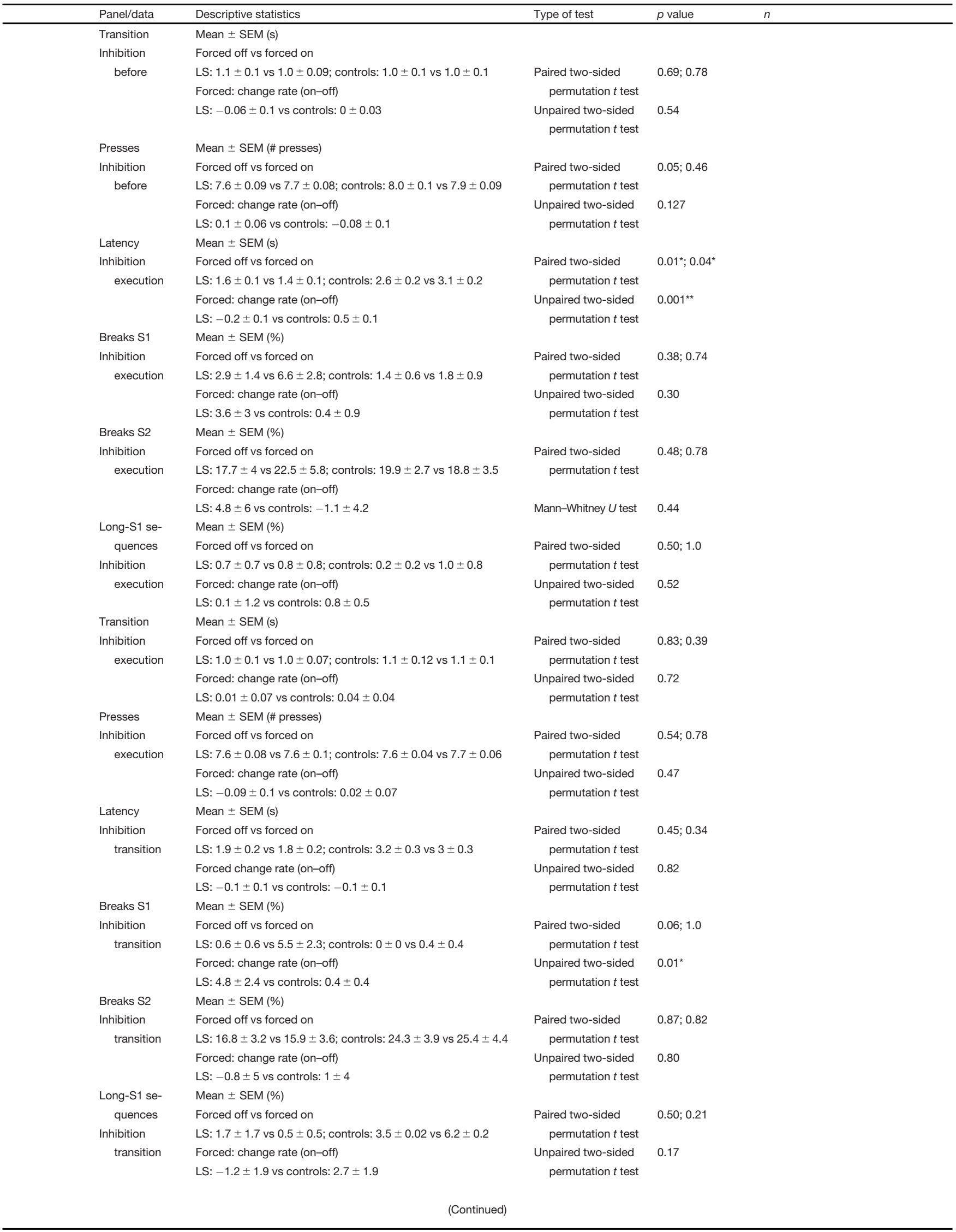


Table 1: Continued

\begin{tabular}{|c|c|c|c|c|c|}
\hline & Panel/data & Descriptive statistics & Type of test & $p$ value & $n$ \\
\hline & Transition & Mean \pm SEM (s) & & & \\
\hline & Inhibition & Forced off vs forced on & Paired two-sided & $0.02^{\star} ; 0.16$ & \\
\hline & transition & LS: $0.8 \pm 0.07$ vs $0.9 \pm 0.08 ;$ controls: $1.2 \pm 0.1$ vs $1.2 \pm 0.1$ & permutation $t$ test & & \\
\hline & & Forced: change rate (on-off) & Unpaired two-sided & $0.0006^{\star \star \star}$ & \\
\hline & & LS: $0.1 \pm 0.04$ vs controls: $-0.03 \pm 0.02$ & permutation $t$ test & & \\
\hline & Presses & Mean \pm SEM (\# presses) & & & \\
\hline & Inhibition & Forced off vs forced on & Paired two-sided & $0.16 ; 0.41$ & \\
\hline & transition & LS: $7.7 \pm 0.04$ vs $7.5 \pm 0.1$; controls: $7.8 \pm 0.1$ vs $7.9 \pm 0.1$ & permutation $t$ test & & \\
\hline & & Forced: change rate (on-off) & Unpaired two-sided & 0.18 & \\
\hline & & LS: $-0.1 \pm 0.1$ vs controls: $0.1 \pm 0.1$ & permutation $t$ test & & \\
\hline \multirow{36}{*}{$\begin{array}{l}\text { Data related to } \\
\text { Figure } 5\end{array}$} & Breaks S1 & Mean \pm SEM (\%) & & & \\
\hline & & Forced off vs forced on & & & \\
\hline & & M2-LS: $0 \pm 0$ vs $0 \pm 0$; M1-LS: $1.3 \pm 0.9$ vs $1.2 \pm 0.8$; controls: & Paired two-sided & $1.0 ; 0.7 ; 0.5$ & Emx1-cre mice $(+/-)$ \\
\hline & & $0.8 \pm 0.5$ vs $0.8 \pm 0.8$ & permutation $t$ test & & AAV-Arch-M2-LS: $n=9$ \\
\hline & & Forced: change rate (on-off) & & & \\
\hline & & M2-LS: $0 \pm 0$ vs controls: $0.05 \pm 0.6$ & Unpaired two-sided & 0.5 & AAV-Arch-M1-LS: $n=8$ \\
\hline & & M1-LS: $-0.1 \pm 1.1$ vs controls: $0.05 \pm 0.6$ & permutation $t$ test & 0.9 & AAV-eYFP-M2-LS: $n=9$ (controls) \\
\hline & Breaks S2 & Mean \pm SEM $(\%)$ & & & \\
\hline & & Forced off vs forced on & & & \\
\hline & & $\begin{array}{l}\text { M2-LS: } 15 \pm 5 \text { vs } 16 \pm 3 ; \text { M1-LS: } 18.9 \pm 3.6 \text { vs } 15.9 \pm 3.5 \text {; con- } \\
\quad \text { trols: } 20 \pm 3.8 \text { vs } 21 \pm 5\end{array}$ & $\begin{array}{l}\text { Paired two-sided } \\
\text { permutation } t \text { test }\end{array}$ & $0.8 ; 0.6 ; 0.9$ & \\
\hline & & Forced: change rate (on-off) & & & \\
\hline & & M2-LS: $0.6 \pm 4$ vs controls: $0.6 \pm 6$ & Unpaired two-sided & 1.0 & \\
\hline & & M1-LS: $-3 \pm 5$ vs controls: $0.6 \pm 6$ & permutation $t$ test & 0.6 & \\
\hline & Sequence & Mean \pm SEM (\# presses) & & & \\
\hline & & Forced off vs forced on & & & \\
\hline & & $\begin{array}{l}\text { M2-LS: } 8.0 \pm 0.2 \text { vs } 8.0 \pm 0.1 ; \text { M1-LS: } 7.7 \pm 0.1 \text { vs } 7.8 \pm 0.1 \\
\quad \text { controls: } 7.7 \pm 0.1 \text { vs } 7.6 \pm 0.09\end{array}$ & $\begin{array}{l}\text { Paired two-sided } \\
\text { permutation } t \text { test }\end{array}$ & $0.43 ; 0.67 ; 0.33$ & \\
\hline & & Forced: change rate (on-off) & & & \\
\hline & & M2-LS: $0.07 \pm 0.08$ vs controls: $-0.1 \pm 0.1$ & Unpaired two-sided & 0.19 & \\
\hline & & M1-LS: $0.04 \pm 0.9$ vs controls: $-0.1 \pm 0.1$ & permutation $t$ test & 0.29 & \\
\hline & Long-S1 & Mean \pm SEM $(\%)$ & & & \\
\hline & sequences & Forced off vs forced on & & & \\
\hline & & $\begin{array}{l}\text { M2-LS: } 4.2 \pm 3.6 \text { vs } 3.1 \pm 2.3 ; \text { M1-LS: } 4.5 \pm 1.9 \text { vs } 1.7 \pm 1.2 \\
\text { controls: } 2.6 \pm 2.6 \text { vs } 2.6 \pm 2\end{array}$ & $\begin{array}{l}\text { Paired two-sided } \\
\text { permutation } t \text { test }\end{array}$ & $0.5 ; 1.0 ; 1.0$ & \\
\hline & & Forced: change rate (on-off) & & & \\
\hline & & M2-LS: $-1 \pm 1.7$ vs controls: $0.9 \pm 0.02$ & Unpaired two-sided & 0.63 & \\
\hline & & M1-LS: $-2.8 \pm 1.2$ vs controls: $0 \pm 0.8$ & permutation $t$ test & 0.05 & \\
\hline & Duration & Mean \pm SEM (s) & & & \\
\hline & & Forced off vs forced on & & & \\
\hline & & $\begin{array}{l}\text { M2-LS: } 5.19 \pm 0.7 \text { vs } 4.7 \pm 0.5 ; \text { M1-LS: } 4.1 \pm 0.4 \text { vs } 4.22 \pm 0.5 \\
\quad \text { controls: } 4.3 \pm 1.0 \text { vs } 3.9 \pm 0.6\end{array}$ & $\begin{array}{l}\text { Paired two-sided } \\
\text { permutation } t \text { test }\end{array}$ & $0.33 ; 0.91 ; 0.47$ & \\
\hline & & Self-paced off vs self-paced on & & & \\
\hline & & $\begin{array}{l}\text { M2-LS: } 6.1 \pm 0.7 \text { vs } 7.3 \pm 1.5 ; \text { M1-LS: } 6.0 \pm 0.5 \text { vs } 6.0 \pm 0.4 \\
\quad \text { controls: } 5.8 \pm 1.2 \text { vs } 5.1 \pm 0.9\end{array}$ & & $0.50 ; 0.82 ; 0.09$ & \\
\hline & & Forced: change rate (on-off) & Unpaired two-sided & $0.91 ; 0.44$ & \\
\hline & & M2-LS: $-0.4 \pm 0.4$ vs controls: $-0.4 \pm 0.4$ & permutation $t$ test & & \\
\hline & & M1-LS: $0.03 \pm 0.4$ vs controls: $-0.4 \pm 0.5$ & & $0.18 ; 0.20$ & \\
\hline & & Self-paced: change rate (on-off) & & & \\
\hline & & M2-LS: $1.15 \pm 1.2$ vs controls: $1.0 \pm 0.3$ & & & \\
\hline & & M1-LS: $-0.05 \pm 0.2$ vs controls: $1.0 \pm 0.3$ & & & \\
\hline \multirow{8}{*}{$\begin{array}{l}\text { Data related to } \\
\text { Figure } 6\end{array}$} & Transition & Mean \pm SEM (s) & & & \\
\hline & & Forced off vs forced on & & & \\
\hline & & M2-LS: $1.0 \pm 0.1$ vs $1.0 \pm 0.1 ;$ M1-LS: $1.2 \pm 0.2$ vs $1.1 \pm 0.1$ & Paired two-sided & $0.21 ; 0.55 ; 0.8$ & Emx1-cre mice (+/-) \\
\hline & & controls: $0.9 \pm 0.1$ vs $0.9 \pm 0.1$ & permutation $t$ test & & AAV-Arch-M2-LS: $n=9$ \\
\hline & & Forced: change rate (on-off) & & & AAV-Arch-M1-LS: $n=8$ \\
\hline & & M2-LS: $-0.04 \pm 0.03$ vs controls: $0 \pm 0.02$ & Unpaired two-sided & 0.34 & AAV-eYFP-M2-LS: $n=9$ (controls) \\
\hline & & M1-LS: $-0.1 \pm 0.14$ vs controls: $0 \pm 0.02$ & permutation $t$ test & 0.59 & \\
\hline & & (Continued) & & & \\
\hline
\end{tabular}


Table 1: Continued

\begin{tabular}{|c|c|c|c|c|}
\hline Panel/data & Descriptive statistics & Type of test & $p$ value & $n$ \\
\hline \multirow[t]{6}{*}{ Breaks S1 } & Mean \pm SEM (\%) & & & \\
\hline & Forced off vs forced on & Paired two-sided & $1.0 ; 1.0 ; 1.0$ & \\
\hline & $\begin{array}{l}\text { M2-LS: } 0 \pm 0 \text { vs } 0 \pm 0 ; \text { M1-LS: } 2.1 \pm 1 \text { vs } 0 \pm 0 ; \text { controls: } 0 \pm 0 \text { vs } \\
\quad 0 \pm 0\end{array}$ & permutation $t$ test & & \\
\hline & Forced: change rate (on-off) & & & \\
\hline & M2-LS: $0 \pm 0$ vs controls: $0 \pm 0$ & Unpaired two-sided & 1.0 & \\
\hline & M1-LS: $-2.1 \pm 1$ vs controls: $0 \pm 0$ & permutation $t$ test & 1.0 & \\
\hline \multirow[t]{6}{*}{ Breaks S2 } & Mean \pm SEM $(\%)$ & & & \\
\hline & Forced off vs forced on & & & \\
\hline & $\begin{array}{l}\text { M2-LS: } 15 \pm 4.6 \text { vs } 18 \pm 4.4 ; \text { M1-LS: } 18 \pm 4.8 \text { vs } 12 \pm 3 \text {; con- } \\
\quad \text { trols: } 24 \pm 5 \text { vs } 22 \pm 3\end{array}$ & $\begin{array}{l}\text { Paired two-sided } \\
\text { permutation } t \text { test }\end{array}$ & $0.49 ; 0.24 ; 0.70$ & \\
\hline & Forced: change rate (on-off) & & & \\
\hline & M2-LS: $2.7 \pm 4$ vs controls: $-1.7 \pm 5$ & Unpaired two-sided & 0.48 & \\
\hline & M1-LS: $-5.9 \pm 4$ vs controls: $-1.7 \pm 5$ & permutation $t$ test & 0.55 & \\
\hline \multirow[t]{11}{*}{ Duration } & Mean \pm SEM $(s)$ & & & \\
\hline & Forced off vs forced on & Paired two-sided & $0.12 ; 0.46 ; 0.07$ & \\
\hline & $\begin{array}{l}\text { M2-LS: } 4.8 \pm 0.3 \text { vs } 4.1 \pm 0.3 ; \text { M1-LS: } 3.8 \pm 0.4 \text { vs } 3.9 \pm 0.4 \\
\quad \text { controls: } 3.4 \pm 0.4 \text { vs } 3.8 \pm 0.4\end{array}$ & permutation $t$ test & & \\
\hline & Self-paced off vs self-paced on & & $0.23 ; 0.69 ; 0.08$ & \\
\hline & $\begin{array}{l}\text { M2-LS: } 5.3 \pm 0.5 \text { vs } 7.7 \pm 1.6 ; \text { M1-LS: } 6.2 \pm 0.6 \text { vs } 6.1 \pm 0.5 \\
\quad \text { controls: } 4.8 \pm 0.6 \text { vs } 4.5 \pm 0.5\end{array}$ & & & \\
\hline & Forced: change rate (on-off) & Unpaired two-sided & $0.04^{\star} ; 0.52$ & \\
\hline & M2-LS: $-0.7 \pm 0.4$ vs controls: $0.3 \pm 0.2$ & permutation $t$ test & & \\
\hline & M1-LS: $0.08 \pm 0.1$ vs controls: $0.3 \pm 0.2$ & & & \\
\hline & Self-paced: change rate (on-off) & & $0.14 ; 0.57$ & \\
\hline & M2-LS: $2.4 \pm 1.7$ vs controls: $-0.2 \pm 0.1$ & & & \\
\hline & M1-LS: $-0.08 \pm 0.2$ vs controls: $-0.2 \pm 0.1$ & & & \\
\hline \multirow{12}{*}{$\begin{array}{l}\text { \# Presses } \\
\text { sequences }\end{array}$} & Mean \pm SEM (\# presses) & & & \\
\hline & Forced off vs forced on & & & \\
\hline & $\begin{array}{l}\text { M2-LS: } 7.9 \pm 0.2 \text { vs } 7.7 \pm 0.07 ; \text { M1-LS: } 7.7 \pm 0.07 \text { vs } \\
\quad 7.8 \pm 0.05 ; \text { controls: } 7.8 \pm 0.2 \text { vs } 8.0 \pm 0.2\end{array}$ & $\begin{array}{l}\text { Paired two-sided } \\
\text { permutation } t \text { test }\end{array}$ & $0.14 ; 0.67 ; 0.19$ & \\
\hline & Self-paced off vs self-paced on & & & \\
\hline & M2-LS: $10.9 \pm 0.6$ vs $11.9 \pm 0.7 ;$ M1-LS: $11.8 \pm 0.7$ vs & & $0.27 ; 0.97 ; 0.25$ & \\
\hline & $11.8 \pm 0.5 ;$ controls: $11.1 \pm 0.3$ vs $10.7 \pm 0.4$ & & & \\
\hline & Forced: change rate (on-off) & & & \\
\hline & M2-LS: $-0.2 \pm 0.1$ vs controls: $0.1 \pm 0.1$ & Unpaired two-sided & $0.049^{*} ; 0.55$ & \\
\hline & M1-LS: $0.05 \pm 0.08$ vs controls: $0.1 \pm 0.1$ & permutation $t$ test & & \\
\hline & Self-paced: change rate (on-off) & & & \\
\hline & M2-LS: $1.0 \pm 0.8$ vs controls: $-0.3 \pm 0.3$ & & $0.11 ; 0.37$ & \\
\hline & M1-LS: $0.01 \pm 0.2$ vs controls: $-0.3 \pm 0.3$ & & & \\
\hline
\end{tabular}

this last difference is that $\mathrm{M} 2 \rightarrow \mathrm{LS}$ activity may be required to set the execution of sequences by impinging on specific striatal subcircuits (Wall et al., 2013), However, our direct inhibition of the LS did not differentiate between striatal subcircuits. To address this, Extended Data Figure 4-3 shows that the M2 $\rightarrow$ LS innervates, strongly the striatal projection neurons from the direct pathway. Such preferential innervation has been previously suggested (Wall et al., 2013; Nelson et al., 2020) to direct the structuring of sequences in serial order tasks (Rothwell et al., 2015), although only after learning.

Lastly, the quantification of the cells that project from M2/M1 to other brain areas by crossing through the LS showed it to be a small percentage of neurons (M2/ $\mathrm{M} 1 \rightarrow \mathrm{Str} \rightarrow \mathrm{Th} 3 \%$; M2/M1 $\rightarrow$ Str $\rightarrow$ Pns 3\%; Extended Data Fig. 4-2). This data, plus the evidence that Arch3.0 mainly has inhibitory actions through local inhibition rather than affecting the propagation of action potentials (El-Gaby et al., 2016), suggests that the effects observed here were predominantly from optogenetic inhibition of the corticostriatal projections from M2 or M1 into the LS.

In conclusion, this study shows that the premotor cortico-striatal projections to the LS contribute to the initiation and execution of self-paced sequences. It supports a model in which both M2 and M1 contain activity modulated by sequence initiation and execution. However, the M2 $\rightarrow$ LS cortico-striatal projections mainly contribute to the proper execution/structuring of self-paced sequences. Both, M2 and M1 cortico-striatal projections contribute to the initiation of sequences. Also, our findings support the idea that the premotor cortico-striatal projections to the LS are a serial driver for the execution of sequences (Lashley, 1951; Zeigler and Gallistel, 1981; Graybiel, 1998; Rosenbaum et al., 2007; Jin and Costa, 2015). Altogether the presented findings may have important implications for pathophysiological conditions whereby self-paced generation of actions is impaired. 


\section{References}

Bartolo R, Merchant $\mathrm{H}$ (2015) $\beta$ Oscillations are linked to the initiation of sensory-cued movement sequences and the internal guidance of regular tapping in the monkey. J Neurosci 35:4635-4640.

Bartolo R, Prado L, Merchant H (2014) Information processing in the primate basal ganglia during sensory-guided and internally driven rhythmic tapping. J Neurosci 34:3910-3923.

Berlot E, Popp NJ, Grafton ST, Diedrichsen J (2021) Combining repetition suppression and pattern analysis provides new insights into the role of $\mathrm{M} 1$ and parietal areas in skilled sequential actions. $J$ Neurosci. Advance online publication. Retrieved Jul 26, 2021. doi: 10.1523/JNEUROSCI.0863-21.2021.

Burguière E, Monteiro P, Feng G, Graybiel AM (2013) Optogenetic stimulation of lateral orbitofronto-striatal pathway suppresses compulsive behaviors. Science 340:1243-1246.

Chen PY, Popovich PM (2011) Correlation: parametric and nonparametric measures. Beverly Hills: Sage University Papers Series on Quantitative Applications in the Social Sciences.

Churchland MM, Santhanam G, Shenoy KV (2006) Preparatory activity in premotor and motor cortex reflects the speed of the upcoming reach. J Neurophysiol 96:3130-3146.

Crowe DA, Zarco W, Bartolo R, Merchant H (2014) Dynamic representation of the temporal and sequential structure of rhythmic movements in the primate medial premotor cortex. J Neurosci 34:11972-11983.

Cui G, Jun SB, Jin X, Pham MD, Vogel SS, Lovinger DM, Costa RM (2013) Concurrent activation of striatal direct and indirect pathways during action initiation. Nature 494:238-242.

Dhawale AK, Wolff SBE, Raymond K, Ölveczky BP (2021) The basal ganglia control the detailed kinematics of learned motor skills. Nat Neuroci 24:1256-1269.

Díaz-Hernández E, Contreras-López R, Sánchez-Fuentes A, RodríguezSibrían L, Ramírez-Jarquín JO, Tecuapetla F (2018) The thalamostriatal projections contribute to the initiation and execution of a sequence of movements. Neuron 100:739-752.e5.

Donoghue JP, Wise SP (1982) The motor cortex of the rat: Cytoarchitecture and microstimulation mapping. J Comp Neurol 212:76-88.

El-Gaby M, Zhang Y, Wolf K, Schwiening CJ, Paulsen O, Shipton OA (2016) Archaerhodopsin selectively and reversibly silences synaptic transmission through altered $\mathrm{pH}$. Cell Rep 16:2259-2268.

Friedman A, Homma D, Gibb LG, Amemori K, Rubin SJ, Hood AS, Riad MH, Graybiel AM (2015) A corticostriatal path targeting striosomes controls decision-making under conflict. Cell 161:13201333.

Fujii N, Graybiel AM (2005) Time-varying covariance of neural activities recorded in striatum and frontal cortex as monkeys perform sequential-saccade tasks. Proc Natl Acad Sci USA 102:90329037.

Geddes CE, Li H, Jin X (2018) Optogenetic editing reveals the hierarchical organization of learned action sequences article optogenetic editing reveals the hierarchical organization of learned action sequences. Cell 174:32-43.

Gorski JA, Talley T, Qiu M, Puelles L, Rubenstein JLR, Jones KR (2002) Cortical excitatory neurons and glia, but not GABAergic neurons, are produced in the Emx1-expressing lineage. $J$ Neurosci 22:6309-6314.

Graybiel AM (1998) The basal ganglia and chunking of action repertoires. Neurobiol Learn Mem 70:119-136.

Guo H, Hong S, Jin XL, Chen RS, Avasthi PP, Tu YT, Ivanco TL, Li Y (2000) Specificity and efficiency of Cre-mediated recombination in Emx1-cre knock-in mice. Biochem Biophys Res Commun 273:661-665.

Hernández A, Nácher V, Luna R, Zainos A, Lemus L, Alvarez M, Vázquez Y, Camarillo L, Romo R (2010) Decoding a perceptual decision process across cortex. Neuron 66:300-314.

Hikosaka O, Isoda M (2010) Switching from automatic to controlled behavior: cortico-basal ganglia mechanisms. Trends Cogn Sci 14:154-161.
Hintiryan H, Foster NN, Bowman I, Bay M, Song MY, Gou L, Yamashita S, Bienkowski MS, Zingg B, Zhu M, Yang XW, Shih JC, Toga AW, Dong HW (2016) The mouse cortico-striatal projectome. Nat Neurosci 19:1100-1114.

Ho J, Tumkaya T, Sameer A, Choi H, Claridge A (2019) Moving beyond $p$ values: data analysis with estimation graphics. Nat Methods 16:565-566.

Hunnicutt BJ, Jongbloets BC, Birdsong WT, Gertz KJ, Zhong H, Mao $\mathrm{T}$ (2016) A comprehensive excitatory input map of the striatum reveals novel functional organization. Elife 5:e19103.

Inase M, Sakai ST, Tanji J (1996) Overlapping corticostriatal projections from the supplementary motor area and the primary motor cortex in the macaque monkey: an anterograde double labeling study. J Comp Neurol 373:283-296.

Jin X, Costa RM (2015) Shaping action sequences in basal ganglia circuits. Curr Opin Neurobiol 33:188-196.

Jin DZ, Fujii N, Graybiel AM (2009) Neural representation of time in cortico-basal ganglia circuits. Proc Natl Acad Sci USA 106:1915619161.

Jin X, Tecuapetla F, Costa RM (2014) Basal ganglia subcircuits distinctively encode the parsing and concatenation of action sequences. Nat Neurosci 17:423-430.

Kawai R, Markman T, Poddar R, Ko R, Fantana AL, Dhawale AK, Kampff AR, Ölveczky BP (2015) Motor cortex is required for learning but not for executing a motor skill. Neuron 86:800-812.

Kobak D, Brendel W, Constantinidis C, Feierstein CE, Kepecs A, Mainen ZF, Qi XL, Romo R, Uchida N, Machens CK (2016) Demixed principal component analysis of neural population data. Elife 5:e10989.

Kupferschmidt DA, Juczewski K, Cui G, Johnson KA, Lovinger DM (2017) Parallel, but dissociable, processing in discrete corticostriatal inputs encodes skill learning. Neuron 96:476-489.e5.

Kurata K, Tanji J (1985) Contrasting neuronal activity in supplementary and precentral motor cortex of monkeys. II Responses to movement triggering vs. nontriggering sensory signals. J Neurophysiol 53:142152.

Lashley K (1951) The problem of serial order in behavior. In: Cerebral mechanisms in behavior (Jeffress LA, ed), pp 112-136. New York: Wiley.

Li N, Daie K, Svoboda K, Druckmann S (2016) Robust neuronal dynamics in premotor cortex during motor planning. Nature 532:459-464.

Lima SQ, Hromádka T, Znamenskiy P, Zador AM (2009) PINP: a new method of tagging neuronal populations for identification during in vivo electrophysiological recording. PLoS One 4:e6099.

Lucchetti C, Bon L (2001) Time-modulated neuronal activity in the premotor cortex of macaque monkeys. Exp Brain Res 141:254260.

Mahn M, Prigge M, Ron S, Levy R, Yizhar O (2016) Biophysical constraints of optogenetic inhibition at presynaptic terminals. Nat Neurosci 19:554-556.

Manouze $\mathrm{H}$, Ghestem A, Poillerat V, Bennis M, Ba-M'hamed S, Benoliel JJ, Becker C, Bernard C (2019) Effects of single cage housing on stress, cognitive, and seizure parameters in the rat and mouse pilocarpine models of epilepsy. eNeuro 6:ENEURO.017918.2019

Martiros N, Burgess AA, Graybiel AM (2018) Inversely active striatal projection neurons and interneurons selectively delimit useful behavioral article inversely active striatal projection neurons and interneurons selectively delimit useful behavioral sequences. Curr Biol 28:560-573.

Mendoza G, Méndez JC, Pérez O, Prado L, Merchant H (2018) Neural basis for categorical boundaries in the primate pre-SMA during relative categorization of time intervals. Nat Commun 9:1098.

Merchant H, Pérez O, Zarco W, Gámez J (2013) Interval tuning in the primate medial premotor cortex as a general timing. $\mathrm{J}$ Neurosci 33:9082-9096. 
Murakami M, Vicente MI, Costa GM, Mainen ZF (2014) Neural antecedents of self-initiated actions in secondary motor cortex. Nat Neurosci 17:1574-1582.

Mushiake H, Inase M, Tanji J (1990) Selective coding of motor sequence in the supplementary motor area of the monkey cerebral cortex. Exp Brain Res 82:208-210.

Nakayama Y, Yamagata T, Tanji J, Hoshi E (2008) Transformation of a virtual action plan into a motor plan in the premotor cortex. $\mathrm{J}$ Neurosci 28:10287-10297.

Nakayama H, Ibañez-Tallon I, Heintz N (2018) Cell-type-specific contributions of medial prefrontal neurons to flexible behaviors. $J$ Neurosci 38:4490-4504.

Nelson A, Abdelmesih B, Costa RM (2020) Corticospinal neurons encode complex motor signals that are broadcast to dichotomous striatal circuits. bioRxiv 2020.08.31.275180.

Oh SW, Harris JA, Ng L, Winslow B, Cain N, Mihalas S, Wang Q, Lau C, Kuan L, Henry AM, Mortrud MT, Ouellette B, Nguyen TN, Sorensen SA, Slaughterbeck CR, Wakeman W, Li Y, Feng D, Ho A, Nicholas E, et al. (2014) A mesoscale connectome of the mouse brain. Nature 508:207-214.

Passingham RE, Myers C, Rawlins N, Lightfoot V, Fearn S (1988) Premotor cortex in the rat. Behav Neurosci 102:101-109.

Redgrave $\mathrm{P}$, Rodriguez M, Smith $\mathrm{Y}$, Rodriguez-Oroz MC, Lehericy S, Bergman H, Agid Y, DeLong MR, Obeso JA (2010) Goal-directed and habitual control in the basal ganglia: implications for Parkinson's disease. Nat Rev Neurosci 11:760-772.

Riehle A, Requin J (1989) Monkey primary motor and premotor cortex: single-cell activity related to prior information about direction and extent of an intended movement. J Neurophysiol 61:534-549.

Riehle A, MacKay WA, Requin J (1994) Are extent and force independent movement parameters? Preparation- and movement-related neuronal activity in the monkey cortex. Exp Brain Res 99:5674.

Romo R, Schultz W (1987) Neuronal activity preceding self-initiated or externally timed arm movement in area 6 of monkey cortex. Exp Brain Res 67:656-602.

Romo R, Schultz W (1992) Role of primate basal ganglia and frontal cortex in the internal generation of movements. Exp Brain Res 91:396-407.

Rosenbaum DA, Cohen RG, Jax SA, Weiss DJ, Van Der Wel R (2007) The problem of serial order in behavior: Lashley's legacy. Hum Mov Sci 26:525-554.
Rothwell PE, Hayton SJ, Sun GL, Fuccillo MV, Lim BK, Malenka RC (2015) Input- and output-specific regulation of serial order performance by corticostriatal circuits. Neuron 88:345-356.

Sakurai Y, Takahashi S, Inoue M (2004) Stimulus duration in working memory is represented by neuronal activity in the monkey prefrontal cortex. Eur J Neurosci 20:1069-1080.

Shima K, Tanji J (2000) Neuronal activity in the supplementary and presupplementary motor areas for temporal organization of multiple movements neuronal activity in the supplementary and presupplementary motor areas for temporal organization of multiple movements. J Neurophysiol 84:2148-2160.

Shima K, Isoda M, Mushiake H, Tanji J (2007) Categorization of behavioural sequences in the prefrontal cortex. Nature 445:315-318.

Siniscalchi MJ, Phoumthipphavong V, Ali F, Lozano M, Kwan AC (2016) Fast and slow transitions in frontal ensemble activity during flexible sensorimotor behavior. Nat Neurosci 19:1234-1242.

Smith KS, Graybiel AM (2013) A dual operator view of habitual behavior reflecting cortical and striatal dynamics. Neuron 79:361374.

Sohur US, Padmanabhan HK, Kotchetkov IS, Menezes JRL, Macklis JD (2014) Anatomic and molecular development of corticostriatal projection neurons in mice. Cereb Cortex 24:293-303.

Storey JD (2002) A direct approach to false discovery rates. J R Stat Soc Series B Stat Methodol 64:479-498.

Tanji J, Evarts EV (1976) Anticipatory activity of motor cortex neurons in relation to direction of an intended movement. J Neurophysiol 39:1062-1068.

Tecuapetla F, Jin X, Lima SQ, Costa RM (2016) Complementary contributions of striatal projection pathways to action initiation and execution. Cell 166:703-715.

Thaler DE, Rolls ET, Passingham RE (1988) Neuronal activity of the supplementary motor area (SMA) during internally and externally triggered wrist movements. Neurosci Lett 93:264-269.

Wall NR, De La Parra M, Callaway EM, Kreitzer AC (2013) Differential innervation of direct- and indirect-pathway striatal projection neurons. Neuron 79:347-360.

Wilson CJ (1989) The basal ganglia. In: The synaptic organization of the brain, Ed 3 (Shepherd GM, ed), pp 279-316. Oxford: Oxford University Press.

Zeigler HP, Gallistel CR (1981) The organization of action: a new synthesis. Am J Psychol 94:190. 